\title{
An investigation of Flow Past a Cylinder Embedded on Curved and Flat Surfaces
}

\author{
Pankaj Jagad and Ravi Samtaney* \\ Mechanical Engineering, Physical Science and Engineering Division, \\ King Abdullah University of Science and Technology, Thuwal, Saudi Arabia \\ Mamdouh S. Mohamed \\ Department of Mechanical Design and Production, \\ Faculty of Engineering, Cairo University, Giza, Egypt
}

(Dated: March 7, 2020)

\begin{abstract}
We investigate the flow past circular, square and triangular cylinders embedded on spherical and cylindrical surfaces and quantify the effect of the embedding surface curvature on the flow characteristics. For the circular cylinder, we examine three Reynolds numbers $R e=40,100$, and 1000 based on cylinder diameter $(D)$, and vary the normalized embedding surface curvature values $\kappa D$, where $\kappa$ is the maximum principal curvature, from 0 (flat surface) to $1 / 12$. Measures of fluid dynamical quantities of interest: drag coefficient $\left(C_{d}\right)$, lift coefficient $\left(C_{l}\right)$, Strouhal number $(S t)$, pressure coefficient $\left(C_{p}\right)$, friction coefficient $\left(C_{f}\right)$, vorticity magnitude $\left(\omega^{*}\right)$, street speed $\left(V_{s t}\right)$, street wavelength $(\lambda)$, length of stationary vortex pair $(L)$, and separation angle $\left(\theta_{\text {sep }}\right)$ show an insignificant effect of the surface curvature. For the range of embedding surface curvature values, the dynamics of the flow past a stationary circular cylinder exhibits near universality, and is essentially independent of the embedding surface curvature. Our conclusion is further consolidated by the similar insensitivity to the embedding surface curvature exhibited by flows past a stationary cylinder having a square or triangular cross-section.
\end{abstract}

\section{INTRODUCTION}

Flows on two-dimensional surfaces are relevant to certain applications such as geophysical flows, atomic physics [1], and bio-membrane flows [2]. Recently, there is a growing interest to explore the effect of geometry and curvature of the underlying surface on the in-surface behavior for various physical applications. The Gaussian curvature effect on the soft materials design was studied in [3]. An investigation of the dynamical deformation of a vesicle membrane in 2-dimensional space showed the effect of the membrane curvature on the membrane deformation [4]. Bowick et al. [5] investigated the underlying surface topology and its Gaussian curvature effects on condensed matter systems. Curvature induces elastic stress in a two-dimensional crystal on the surface of a sphere due to its incompatibility with the crystal axes, which can be relaxed by Asaro-Tiller-Grinfeld (ATG) instability as demonstrated in [6]. For the particle inclusions embedded within curved lipid bilayer membranes, the interplay between curvature and topology can augment hydrodynamic responses for spherical vesicles, resulting in induced shears and recirculation as depicted in [7]. The interplay of the flow field with the topology was demonstrated in [8] by means of realizations of the Poincaré-Hopf theorem on n-tori. According to Nestler et al. [9], for a surface bound systems of densely packed rodlike particles tending to align tangentially, the geometric properties influence the realizations of the Poincaré-Hopf theorem. For the flow of passive and active polar liquid crystals, whose molecular orientation is subjected to a

* Ravi.Samtaney@kaust.edu.sa tangential anchoring on an evolving curved surface, the effect of the underlying curvature on the location of topological defects on a torus was demonstrated in [10]. The surface curvature effect on the vortex dynamics for flows on curved surfaces has also been reported [11-13]. A conformal mapping based formulation permitting the calculation of the energetics of vortices on rigid substrates of spatially varying Gaussian curvature was presented in [11]. The geometric force exerted on vortices in curved superfluid films was shown to attract/repel vortices towards regions of negative/positive Gaussian curvature. The influence of the Gaussian curvature using a surface vorticity-stream function formulation, and parametric finite elements method was demonstrated in [12]. The additional geometric terms in the incompressible surface Navier-Stokes equations induced a strong coupling between topology, geometry, and viscosity. On closed surfaces, the defects in the flow field, whose presence might be because of the topological constraints, responded to the surface geometry and its changes, and interacted with each other. Point vortex interactions on a toroidal surface using a point vortex model and its comparison with the vortex dynamics on a flat torus was studied in [13]. It was found that two identical point vortices can be locally repulsive when small perturbations are added to the initial configuration. A study regarding surface curvature effect on heat transfer rate has also been reported [14]. Their investigation showed that a dimpled concave surface has a higher heat transfer rate than a dimpled flat surface. Whereas, a dimpled convex surface has a lower heat transfer rate than a dimpled flat surface. Studies showing the Gaussian curvature effect on the vortical structures in a cylinder wake have also been reported $[15,16]$. The presence of Gaussian peak and valley in 
the cylinder wake of an incompressible flow brings on some local deformations in the configurations of vortices but does not drastically affect the development of vortex street as reported in [15]. Interplay between geometry, curvature and vorticity dynamics for incompressible flows on curved surfaces was studied in [16]. For the presence of a Gaussian bump in a cylinder wake, the surface geometry significantly affected the vortex street by forming a velocity depression. Curvature generated vorticity and vortical structure evolution was affected by the geometry of the surface.

The aforementioned studies motivate the question concerning how the surface curvature can affect the dynamics of flows past bluff bodies embedded on curved surfaces. Research on the dynamics of flow past a circular cylinder embedded on a flat surface dates back to the century old discovery of Kármán vortex street [17]. Some of the classic studies on this topic include the work by Kármán, Taylor and others [17-21]. The subject of flow past a cylinder is vast and some reviews of the literature on this topic are available in [22-26]. It is evident from the literature that the dynamics of the flow past a cylinder embedded on a flat surface have been extensively studied. To the best of our knowledge, the effect of the embedding surface curvature on the dynamics of flow past a cylinder embedded on curved surfaces has not been investigated yet. The flow past a circular cylinder is a canonical problem in fluid mechanics, and as such an investigation into the effect of the embedding geometry's curvature is of academic interest. On the other hand, such a research problem is not of interest only from an academic perspective but also for applications related to geophysical flows (for example, ocean currents past islands etc.). While some previous research have reported significant surface curvature effects on the dynamics of certain types of flows, this work explores such effects for a different and practically important flow type. Moreover, experimentally, an innovative technique to investigate the dynamics of two-dimensional (2D) flows past a cylinder involves the use of soap-films [27-32]. We note that the soap-film experimental setup may also be suitable to explore the dynamics of surface flows past a cylinder embedded on curved surfaces. Numerical investigations of such flows are precursors to potential future experimental investigations.

In this paper, we study the dynamics of flows past a stationary circular, square, and triangular cylinder embedded on spherical and cylindrical surfaces using a discrete exterior calculus (DEC) scheme [33]. The radius/curvature of the embedding surface is varied and its effect on the flow dynamics is evaluated at different Reynolds numbers. The paper outline is as follows. A description of the physical setup and computational domain for all simulation cases is presented first. The simulations results are subsequently presented along with detailed quantification of the important fluid dynamical measures. The paper closes by emphasizing the key conclusions deduced from the conducted simulations. A brief overview of DEC is presented in Appendix A, followed by a concise discussion of the employed numerical scheme in Appendix B.

\section{PHYSICAL SETUP AND COMPUTATIONAL DOMAIN}

Schematics of the physical setup and the computational domain are shown in figures $1 \mathrm{a}$ and $1 \mathrm{~b}$ for the cases of spherical and cylindrical embedding surfaces, respectively. The circular cylinder contour embedded on the curved surfaces is generated by intersecting a cylinder of diameter $\mathrm{D}=1$ with the spherical and cylindrical surfaces. In terms of geodesic distances, the domain lengths upstream and downstream of the cylinder are 10D and $60 \mathrm{D}$, respectively. The domain width on either side of the cylinder is $10 \mathrm{D}$. These domain sizes are typical in computational investigations of flow past a cylinder on a flat surface. For flows past square and triangular cylinders, computational domains similar to that for the circular cylinder are used except that the cylindrical and/or spherical surfaces are instead intersected with square and triangular prisms, respectively. The implemented boundary conditions are shown in figure 1 for each domain boundary, where $u$ and $v$ for a specific boundary represent the local normal and tangential velocity components. The imposed boundary conditions create flows on a sector of a spherical or cylindrical shape. The present flow configuration was chosen by design to facilitate comparison with a similar configuration of flow past a cylinder on a planar strip. Here, in passing, we note that the present setup limits the maximum curvature of the embedding surface, with higher curvature resulting in the outflow boundary getting wrapped over the inflow boundary. Theoretically, with DEC it is possible to include a cut in the domain and wrap a portion of the domain onto itself. We will explore the numerical implementation of this in the future.

\section{RESULTS AND DISCUSSION}

\section{A. Circular Cylinder}

The computational domain is discretized using a triangular mesh with locally refined mesh close to the cylinder and in the wake. The number of mesh nodes and elements are about $80 \mathrm{k}$ and $160 \mathrm{k}$, respectively. We present simulation results for the flow past a circular cylinder with the configurations corresponding to (i) spherical embedding surfaces and (ii) cylindrical embedding surfaces, both with surface radii $\mathrm{R}=12,18,24,30,36$, and 72 , and (iii) a flat embedding surface as a reference. Reynolds numbers $\left(R e=\rho u_{\infty} D / \mu\right)$ of 40,100 , and 1000 are considered for all test cases. Here, $\rho$ and $u_{\infty}$ denote the fluid density (taken to be unity) and the free stream velocity at the inlet, respectively. The following subsections 


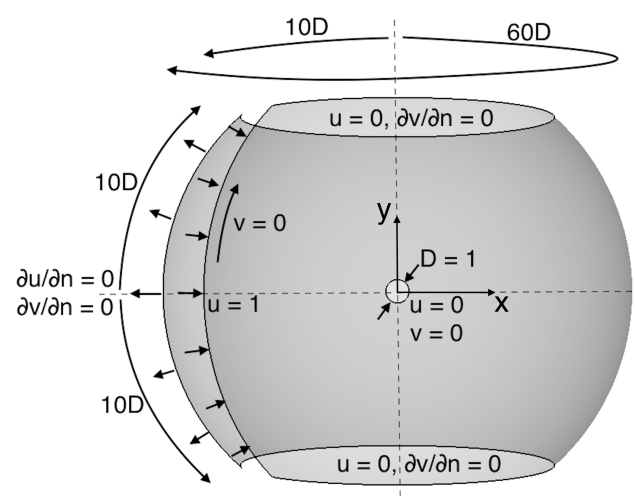

u: normal velocity, 'v: tangential velocity

(a) Spherical surface

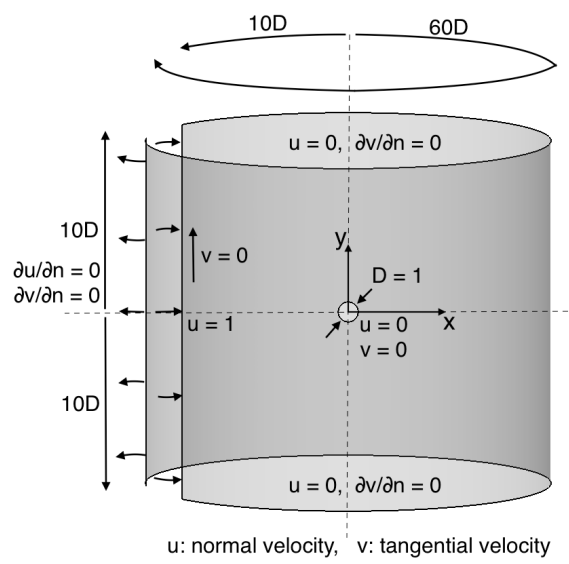

(b) Cylindrical surface

FIG. 1: Schematic of the computational domain for both spherical and cylindrical surfaces showing the domain dimensions and the boundary conditions. Velocity components $(u, v)$ indicate the local normal and tangential velocity components on each boundary.

demonstrate the surface curvature effects on various flow characteristics.

\section{Wake Vortices}

Figures 2a, 2b, and 2c show the Kármán vortex street in the circular cylinder wake for different radii of the cylindrical and spherical embedding surfaces, in addition to the flat embedding surface as a reference. The results are shown for $R e=40,100$, and 1000. For both curved and flat embedding surfaces, there is a stationary pair of vortices attached to the cylinder for $R e=40$, and vortex shedding for $R e=100$ and 1000. Qualitatively, the vortices for the curved embedding surfaces are similar in size and shape to those for the flat embedding surface at each Reynolds number. Quantitative comparisons are presented in ensuing sections.

\section{Drag Coefficient, Lift Coefficient, and Strouhal Number}

The net force acting on the cylinder, due to pressure and viscous forces, is expressed as [34]

$$
\vec{F}=\int_{S}-p \vec{n} d A+\mu \int_{S} \vec{\omega} \times \vec{n} d A,
$$

where $p$ is the static pressure, $\vec{n}$ is the unit normal facing outward of the cylinder, $\vec{\omega}$ is the vorticity vector, $d A$ is an infinitesimal area (infinitesimal length here), and $S$ denotes for the total surface area (perimeter here) of the cylinder. Since the boundary corresponding to the cylinder wall is discretized with $N$ mesh edges, the discrete expression of the force vector is

$$
\vec{F}=\sum_{k=1}^{N}-p_{k} \vec{n}_{k} l_{k}+\mu \sum_{k=1}^{N} l_{k} \vec{\omega}_{k} \times \vec{n}_{k}
$$

where the summation is over the cylinder boundary edges. Here, we consider the global $(x, y, z)$ coordinate system with the cylinder axis intersection with the embedding surface as the origin. The z-direction is along the cylinder axis, and the $\mathrm{x}$ and $\mathrm{y}$ directions are as shown in figure 1 . The pressure $p_{k}$ is the static pressure in the triangles adjacent to the boundary edges, $\vec{n}_{k}$ is the unit vector normal to the edge (projected on the $\mathrm{x}-\mathrm{y}$ plane), and $l_{k}$ is the edge length. For the case of $2 \mathrm{D}$ surfaces, the vorticity is calculated as a scalar on the primal mesh nodes [33]. The vorticity vector is $\vec{\omega}_{k}=\vec{n}_{k, s}\left|\omega_{k}\right|$, with $\vec{n}_{k, s}$ denotes the unit normal to the embedding surface facing outward at the boundary edge midpoint. The vorticity magnitude $\left|\omega_{k}\right|$ is calculated by averaging the vorticity on both of the edge nodes. The drag force $F_{d}$ and the lift force $F_{l}$ are respectively the $x$ and $y$ components of the force vector $\vec{F}$. The drag and lift coefficients are calculated as $C_{d}=F_{d} /\left(\frac{1}{2} \rho u_{\infty}^{2} D\right)$, and $C_{l}=F_{l} /\left(\frac{1}{2} \rho u_{\infty}^{2} D\right)$, respectively. The Strouhal number is $S t=f D / u_{\infty}$, where $f$ is the vortex shedding frequency.

For different embedding surfaces, overlapping plots of the drag coefficient $C_{d}$ as a function of time are shown in figures $3 \mathrm{a}$ and $3 \mathrm{~b}$ for $R e=100$ and 1000, respectively. The shown results are for a finite time duration after the periodically steady vortex shedding commences. Here, the phases for the curved embedding surfaces are slightly adjusted to match with that for the flat embedding surface. The drag coefficient mean values are reported in table I. For $R e=100$, the mean drag coefficient for the flat embedding surface is 1.386 , which is close to values reported in the literature (e.g., 1.38 (Russell and 

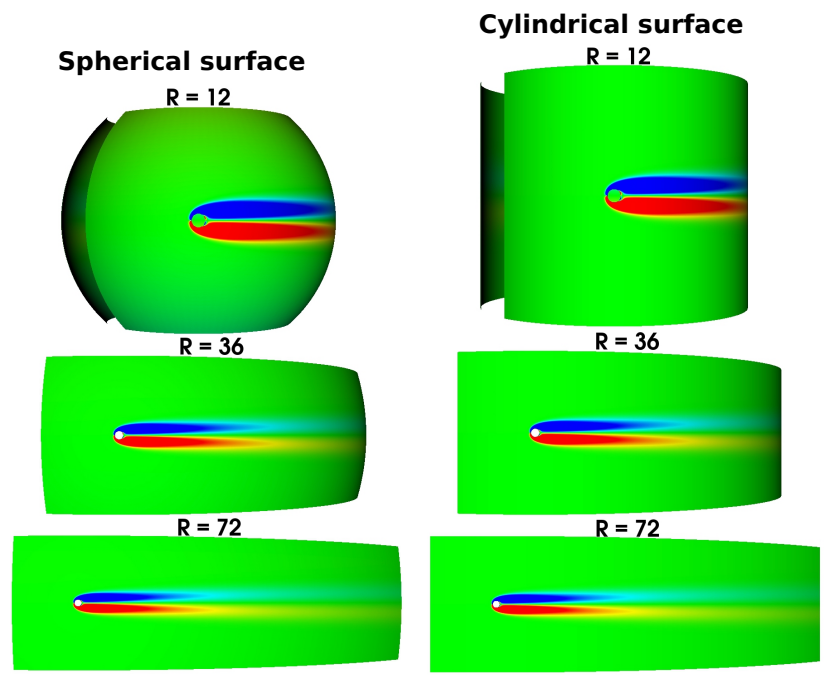

Flat surface

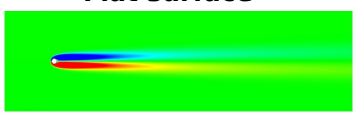

(a) $R e=40$
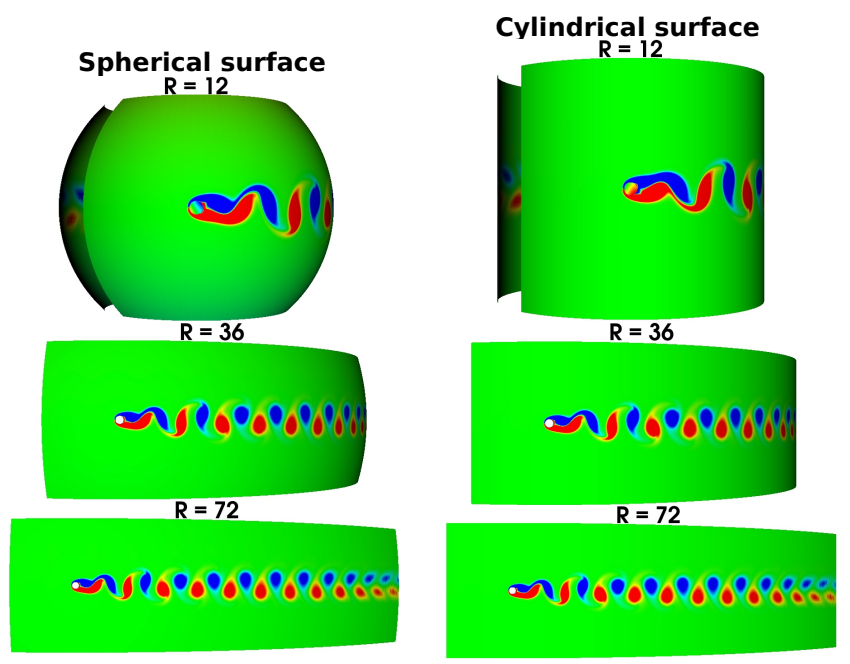

(b) $R e=100$
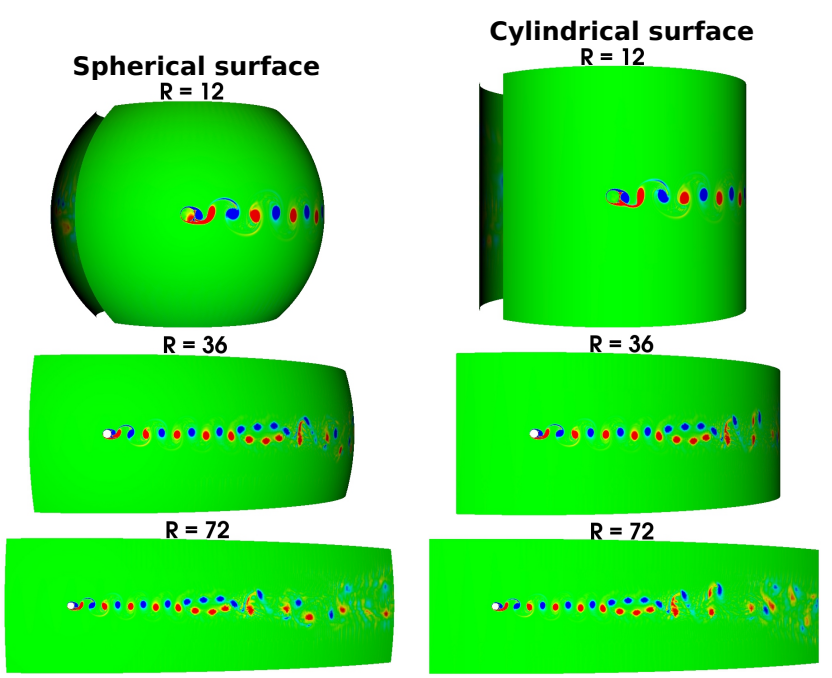

Flat surface

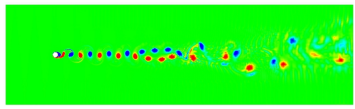

(c) $R e=1000$

FIG. 2: Snapshots of vortex streets for spherical, cylindrical and reference flat embedding surfaces at various $R e$.

Here, $R$ denotes radius of the embedding surface.

Wang[35]), 1.37 (Le et al.[36])). For the case of spherical embedding surface, the mean drag coefficient is 1.386 for $R=72$. The mean drag coefficient decreases slightly with increasing surface curvature (i.e. decreasing spherical surface radius), and for $R=12$ (the highest curvature case) the mean drag coefficient is 1.374 . The change in $C_{d}$ with curvature is almost $1 \%$ and thus very weakly dependent on (or perhaps even independent of) the embedding surface curvature. The mean drag coefficient almost coincides with that for the flat embedding surface. For the case of a cylindrical embedding surface, the mean drag coefficient is constant at 1.386 coinciding with that for the flat embedding surface. Similar analysis for $R e=40$ and 1000 show that the maximum variations in the mean drag coefficient with the embedding surface radius are almost $2 \%$ and $0.5 \%$, respectively, where the mean drag 
coefficient almost coincides with that for the flat embedding surface. Relative changes in the order of $2 \%$ are not unusual during numerical simulations when, for example, the mesh resolution changes or a different quadrature is used. Accordingly, one may reasonably conclude that the effect of the embedding surface curvature on the drag coefficient is insignificant.

Figures $4 \mathrm{a}$, and $4 \mathrm{~b}$ show overlapping plots of the lift coefficient as a function of time at $R e=100$ and 1000, respectively. The phases for the curved embedding surfaces are slightly adjusted to match with that for the flat embedding surface. The RMS values of the lift coefficient are reported in table I. For $R e=100$, the RMS lift coefficient is 0.248 for the flat embedding surface, which is within the range of values reported in the literature (e.g., 0.233 in (Stålberg et al. [37])). The RMS is computed to be 0.248 for the spherical embedding surface having $R=72$, which then decreases slightly with increasing the surface curvature (decreasing surface radius), and is 0.246 for $R=12$, i.e. a negligible decrease (less than $1 \%$ ) in the value. For the cylindrical surface cases, all RMS values are constant at 0.248 coinciding with that for the flat surface. Similarly for $R e=1000$, the maximum variation in the RMS value with the embedding surface curvature is almost $0.6 \%$ and the values almost coincide with that for the flat embedding surface. These results suggest insignificant effect of the embedding surface curvature on the lift coefficient RMS value. It is worth noting that there exists a z-component of the net force on the cylinder due to the viscous term, but this is negligibly small for both the spherical and cylindrical embedding surfaces.

The Strouhal number values are reported in table I for all test cases of flow past a circular cylinder. For the flat embedding surface at $R e=100$, the Strouhal number is computed as 0.171 , which is within the range of values reported in the literature (e.g., 0.166 in (Stålberg et al. $[37])$ ). For the spherical embedding surface, the Strouhal number is 0.171 for $R=72$. It changes negligibly with the surface radius until reaching 0.170 for $R=12$. Thus, it almost coincides with that for the flat embedding surface. The Strouhal number is almost constant at 0.171 irrespective of the surface radius for the cylindrical embedding surface cases, coinciding with that for the flat embedding surface. Similarly at $R e=1000$, the Strouhal number values for the curved embedding surfaces almost coincide with that for the flat embedding surface, irrespective of the embedding surface radius. These results suggest the negligible effect of the embedding surface curvature/radius on the Strouhal number.

3. Distribution of Pressure Coefficient, Skin-Friction Coefficient, and Vorticity Magnitude Along the Cylinder Circumference

The plots of pressure coefficient $C_{p}=$ $\left(p-p_{\infty}\right) /\left(\frac{1}{2} \rho u_{\infty}^{2}\right)$ as a function of the angular position on the cylinder surface $(\theta)$ for all embedding surfaces of different radii, as well as for the flat embedding surface, are shown in figures $5 \mathrm{a}, 5 \mathrm{~b}$ and $5 \mathrm{c}$ at $R e=40, R e=100$, and $R e=1000$, respectively. Here, $p_{\infty}$ denotes the free stream pressure, and time-averaged values of $C_{p}$ are considered. For each of the Reynolds numbers, the plots overlap, which further supports the negligible effect of the embedding surface curvature on the flow dynamics. Similarly results are also observed for the time-averaged skin friction coefficient $C_{f}=\tau_{w} /\left(\frac{1}{2} \rho u_{\infty}^{2}\right)$, and vorticity magnitude $\omega^{*}=\omega_{n} D / u_{\infty}$ as a function of the angular position on the cylinder surface, as shown in figures 6 , and 7. Here, $\tau_{w}$ is the wall shear stress, and $\omega_{n}$ is the vorticity in the surface normal direction (considered as a scalar for calculations on surfaces). The plots indicate the absence of any significant dependence on the embedding surface curvature for all of the Reynolds numbers.

\section{Flow Separation Angle}

The instantaneous flow separation angle values $\theta_{\text {sep }}$ (see figure 8 for the definition) are summarized in table II for all test cases. For $R e=40$, the flow separation angle for the flat embedding surface is $54.75^{\circ}$ and is within the range mentioned in the literature (e.g., Kawaguti and Jain [43], Taneda [44]). The flow separation angles for the spherical and cylindrical embedding surfaces are within almost $\pm 5 \%$ of that for the flat surface. The flow separation angles are $116.55^{\circ}$ and $108.87^{\circ}$ for $R e=100$ and 1000 , respectively, for the flat embedding surface. The reported values in Rajani et al. [42] are $116.55^{\circ}$ (with a periodic variation of $\pm 2.83^{\circ}$ ), and $100.17^{\circ}$ (with a periodic variation of $\pm 18.9^{\circ}$ ) for $R e=100$ and 1000 , respectively. Therefore, the results in table II suggest that the flow separation angle values for the curved embedding surfaces are within the range of values for the flat embedding surface. Accordingly, there is insignificant effect of the embedding surface curvature on the flow separation angle.

\section{Length of Stationary Vortex Pair}

For $R e=40$, there is an attached stationary pair of vortices in the cylinder wake. The length of this vortex pair is defined as the distance between the cylinder center and the end of the stationary vortex (see figure 8a). Values of the stationary vortex pair length are reported in table III for the spherical, cylindrical, and flat embedding surfaces. For the curved embedding surfaces, the lengths are in terms of geodesic distances. The vortex pair length is 2.78 for the flat embedding surface, which is within the range reported in the literature (e.g., Kawaguti and Jain [43], Apelt [45]). The maximum difference in values for the curved embedding surfaces is almost $2 \%$ relative to the flat embedding surface. Thus, there is insignificant 


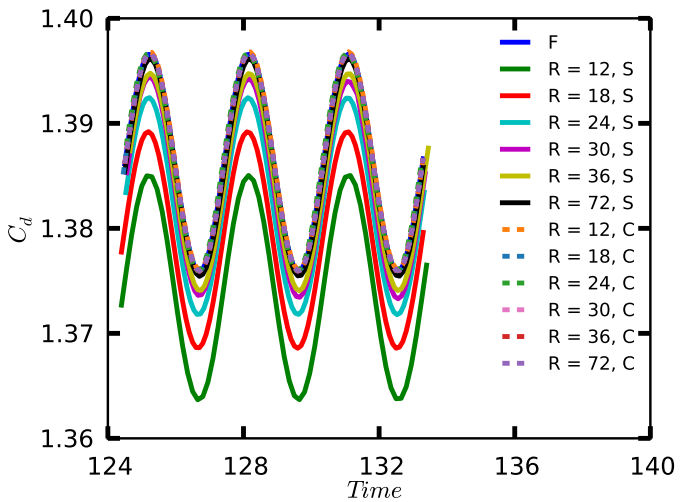

(a) $R e=100$

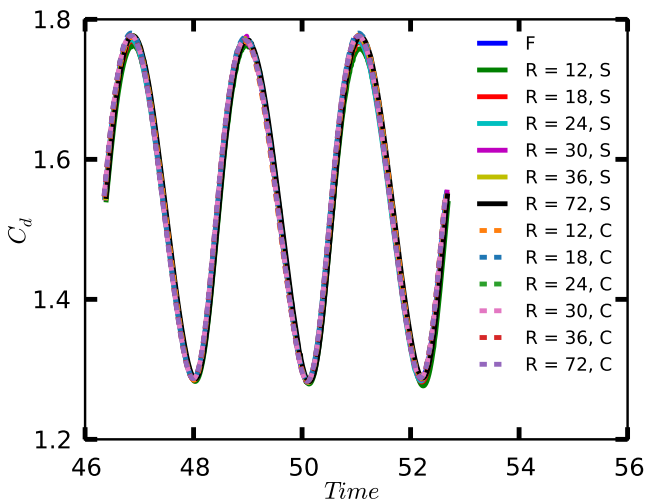

(b) $R e=1000$

FIG. 3: Drag coefficient as a function of time. Here, R: radius of the embedding surface, S: spherical embedding surface, C: cylindrical embedding surface, and F: flat embedding surface.

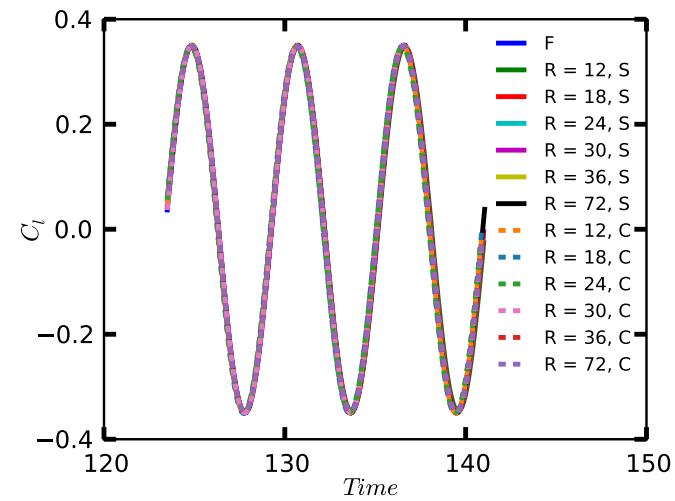

(a) $R e=100$

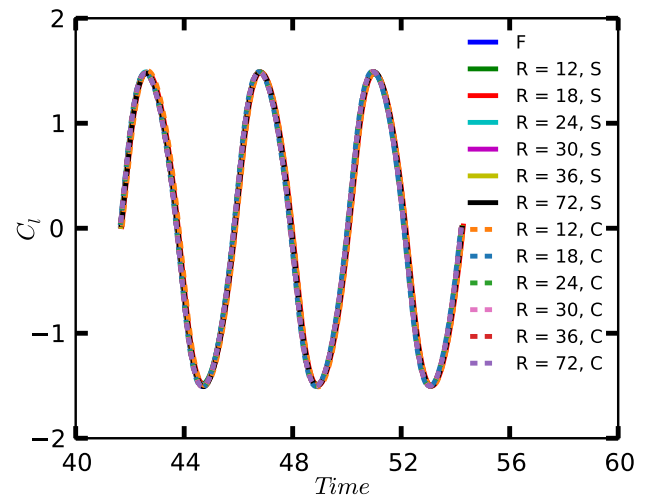

(b) $R e=1000$

FIG. 4: Lift coefficient as a function of time.

effect of the embedding surface curvature on the length of the stationary vortex pair.

\section{Street Speed, and Street Wavelength}

Figure 9 shows the notion of street speed $\left(V_{s t}\right)$ and that of the street wavelength $(\lambda)$. For $R e=100$, after a distance of almost 10D downstream of the cylinder and up to almost 40D, the street speed and wavelength are approximately constant once the flow reaches its quasisteady periodic state. The street speed and wavelength are reported in table IV for $R e=100$. The street speed for a curved embedding surfaces is calculated as the geodesic distance moved by a vortex between two consecutive time instances divided by the time difference between the instances. The street speed is 0.8694 for the flat embedding surface which almost agrees with the experimental value of $0.81 \pm 0.04$ reported in Kim and Wu [46]. The maximum difference for the curved embedding surfaces is $5 \%$ relative to the flat embedding surface. The street wave- length is calculated as the geodesic distance between two consecutive vortices of the same sign. The wavelength is 5.125 for the flat embedding surface, compared with the experimental value of 4.5 reported in Kim and $\mathrm{Wu}$ [46]. The maximum difference for the curved embedding surfaces is almost $2 \%$ relative to the flat embedding surface. Thus, the street speed and wavelength values for the curved embedding surfaces almost coincide with that for the flat embedding surface and there is insignificant effect of the embedding surface curvature on both measures.

\section{B. Square Cylinder}

Flow past a square cylinder of side length $(L)$ unity and oriented to have zero angle of incidence is considered. We present simulation results for configurations corresponding to (i) spherical embedding surfaces and (ii) cylindrical embedding surfaces, both with surface radii $R=12$, and 72 , and (iii) a flat embedding surface as a reference. 


\begin{tabular}{|c|c|c|c|c|c|c|c|c|}
\hline \multirow[t]{2}{*}{ Surface type } & \multirow[t]{2}{*}{ Radius of the surface } & \multirow{2}{*}{$\begin{array}{c}R e=40 \\
C_{d}\end{array}$} & \multicolumn{3}{|c|}{$R e=100$} & \multicolumn{3}{|c|}{$R e=1000$} \\
\hline & & & $C_{d}$ (mean) & $C_{l}(\mathrm{RMS})$ & $S t$ & $C_{d}($ mean $)$ & $C_{l}(\mathrm{RMS})$ & $S t$ \\
\hline \multirow[t]{6}{*}{ Spherical surface } & 12 & 1.571 & 1.374 & 0.246 & 0.17 & 1.536 & 1.075 & 0.238 \\
\hline & 18 & 1.590 & 1.379 & 0.247 & 0.17 & 1.541 & 1.079 & 0.238 \\
\hline & 24 & 1.597 & 1.382 & 0.247 & 0.171 & 1.542 & 1.080 & 0.239 \\
\hline & 30 & 1.599 & 1.384 & 0.248 & 0.171 & 1.542 & 1.080 & 0.239 \\
\hline & 36 & 1.600 & 1.384 & 0.248 & 0.171 & 1.543 & 1.080 & 0.239 \\
\hline & 72 & 1.604 & 1.386 & 0.248 & 0.171 & 1.543 & 1.081 & 0.239 \\
\hline \multirow[t]{6}{*}{ Cylindrical surface } & 12 & 1.605 & 1.386 & 0.248 & 0.171 & 1.544 & 1.081 & 0.239 \\
\hline & 18 & 1.605 & 1.386 & 0.248 & 0.171 & 1.544 & 1.081 & 0.239 \\
\hline & 24 & 1.605 & 1.386 & 0.248 & 0.171 & 1.544 & 1.081 & 0.239 \\
\hline & 30 & 1.605 & 1.386 & 0.248 & 0.171 & 1.544 & 1.081 & 0.239 \\
\hline & 36 & 1.605 & 1.386 & 0.248 & 0.171 & 1.544 & 1.081 & 0.239 \\
\hline & 72 & 1.605 & 1.386 & 0.248 & 0.171 & 1.544 & 1.081 & 0.239 \\
\hline \multirow[t]{9}{*}{ Flat surface } & Present study & 1.605 & 1.386 & 0.248 & 0.171 & 1.544 & 1.081 & 0.239 \\
\hline & Russell and Wang [35] & 1.600 & 1.380 & - & 0.172 & - & - & - \\
\hline & Le et al. $[36]$ & 1.560 & 1.370 & - & 0.160 & - & - & - \\
\hline & Stålberg et al. [37] & 1.53 & 1.32 & 0.233 & 0.166 & - & - & - \\
\hline & Kawaguti [38] & 1.618 & - & - & - & - & - & - \\
\hline & Dennis and Chang [39] & 1.522 & - & - & - & - & - & - \\
\hline & Henderson and Karniadakis [40] & - & - & - & - & 1.5144 & 1.0494 & 0.237 \\
\hline & Sheard et al. $[41]$ & - & - & - & - & 1.500 & - & - \\
\hline & Rajani et al. [42] & - & 1.342 & - & 0.166 & 1.492 & - & 0.244 \\
\hline
\end{tabular}

TABLE I: Mean drag coefficient, RMS lift coefficient, and Strouhal number for all test cases of flow past a circular cylinder.

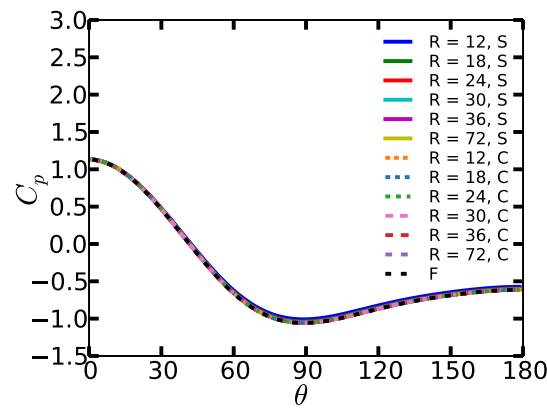

(a) $R e=40$

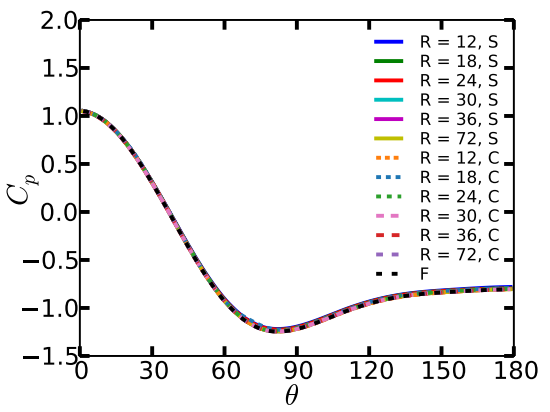

(b) $R e=100$

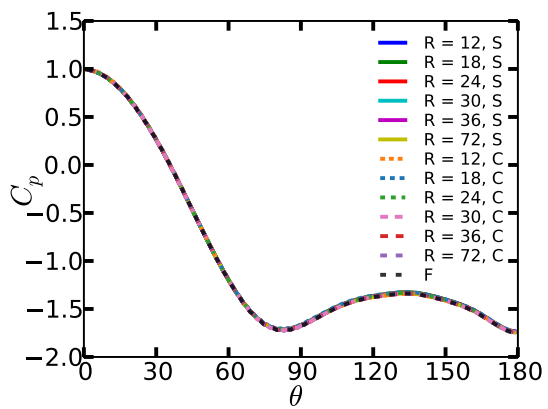

(c) $R e=1000$

FIG. 5: Pressure coefficient as a function of the angular position on the circular cylinder.

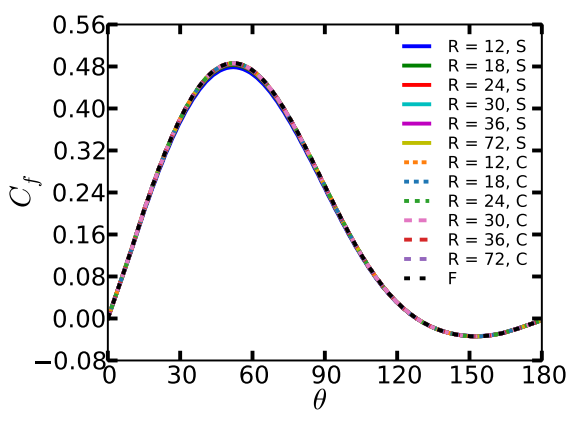

(a) $R e=40$

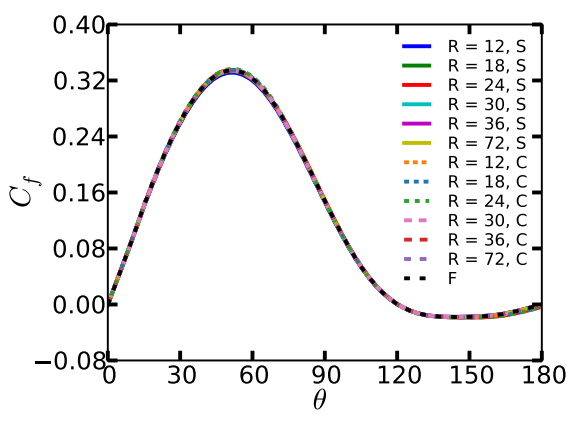

(b) $R e=100$

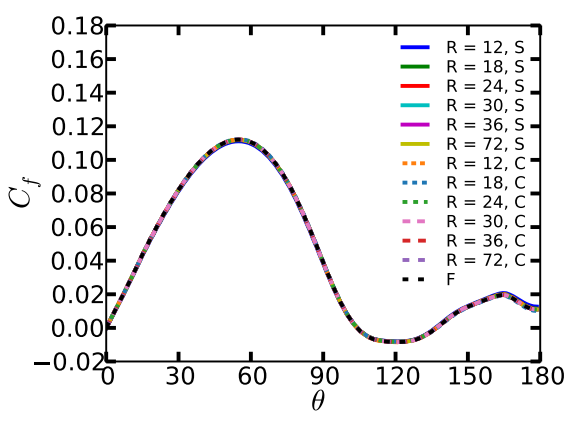

(c) $R e=1000$

FIG. 6: Skin friction coefficient as a function of the angular position on the circular cylinder. 


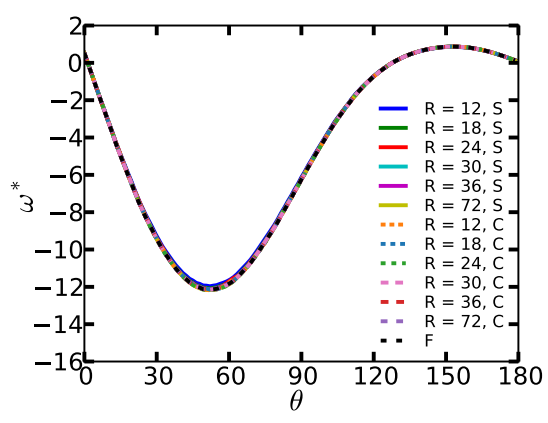

(a) $R e=40$

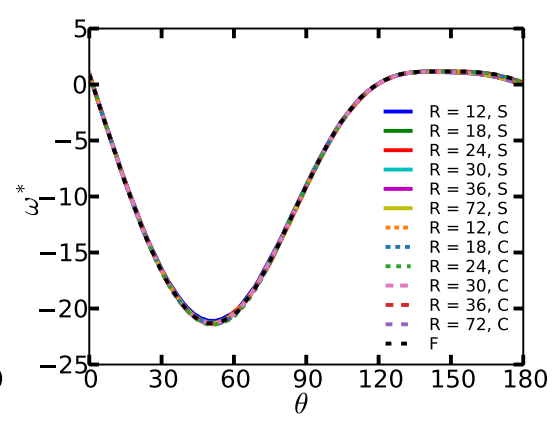

(b) $R e=100$

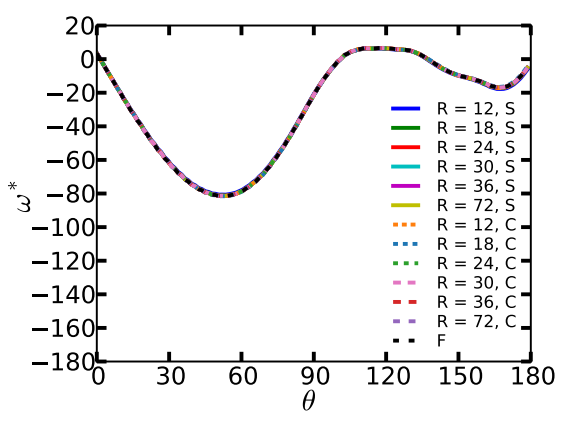

(c) $R e=1000$

FIG. 7: Vorticity magnitude as a function of the angular position on the circular cylinder.

\begin{tabular}{|c|c|c|c|c|}
\hline $\begin{array}{c}\text { Surface } \\
\text { type }\end{array}$ & Radius of the surface & $\begin{array}{c}R e=40 \text { (from downstream } \\
\text { stagnation point) }\end{array}$ & $\begin{array}{c}R e=100 \text { (from } \\
\text { upstream stagnation point) }\end{array}$ & $\begin{array}{c}R e=1000 \text { (from upstream } \\
\text { stagnation point) }\end{array}$ \\
\hline Spherical & 12 & 54.57 & 115.66 & 107.10 \\
\hline surface & 18 & 54.66 & 115.46 & 102.34 \\
\hline & 24 & 54.57 & 116.99 & 106.46 \\
\hline & 30 & 53.45 & 117.00 & 109.12 \\
\hline & 36 & 54.5 & 116.97 & 109.44 \\
\hline & 72 & 54.5 & 115.06 & 109.30 \\
\hline Cylindrical & 12 & 52.24 & 115.46 & 108.67 \\
\hline surface & 18 & 52.97 & 116.51 & 107.67 \\
\hline & 24 & 53.29 & 116.52 & 108.93 \\
\hline & 30 & 52.81 & 116.77 & 108.94 \\
\hline & 36 & 52.88 & 116.55 & 109.11 \\
\hline & 72 & 52.88 & - & 108.87 \\
\hline Flat & Present study & 54.75 & - & - \\
\hline surface & Kawaguti and Jain $[43]$ & 53.7 & $117.05 \pm 2.83$ (periodic & $100.17 \pm 18.9$ (periodic \\
\hline & Taneda [44] & 53.0 & variation) & variation) \\
\hline & Rajani et al. $[42]$ & - & & \\
\hline
\end{tabular}

TABLE II: Flow separation angle values (in degrees) for all flow past a circular cylinder test cases.

\begin{tabular}{|c|c|c|}
\hline Surface type & Radius of the surface & L \\
\hline Spherical surface & 12 & 2.83 \\
\hline & 18 & 2.81 \\
\hline & 24 & 2.83 \\
\hline & 30 & 2.79 \\
\hline & 36 & 2.80 \\
\hline & 72 & 2.80 \\
\hline Cylindrical surface & 12 & 2.78 \\
\hline & 18 & 2.77 \\
\hline & 24 & 2.78 \\
\hline & 30 & 2.77 \\
\hline & 36 & 2.77 \\
\hline & 72 & 2.77 \\
\hline Flat surface & Present study & 2.78 \\
\hline & Kawaguti and Jain [43] & 3.01 \\
\hline & Apelt [45] & 2.64 \\
\hline
\end{tabular}

TABLE III: Length of the stationary vortex pair at $R e=40$ for flow past a circular cylinder. 


\begin{tabular}{|c|c|c|c|}
\hline Surface type & Radius of the surface & $V_{s t} / u_{\infty}$ & $\lambda$ \\
\hline Spherical surface & 12 & 0.9148 & 5.125 \\
\hline & 18 & 0.9146 & 5.122 \\
\hline & 24 & 0.9146 & 5.214 \\
\hline & 30 & 0.9148 & 5.124 \\
\hline & 36 & 0.8702 & 5.216 \\
\hline & 72 & 0.8698 & 5.125 \\
\hline Cylindrical surface & 12 & 0.8694 & 5.125 \\
\hline & 18 & 0.8694 & 5.216 \\
\hline & 24 & 0.8694 & 5.216 \\
\hline & 30 & 0.8694 & 5.125 \\
\hline & 36 & 0.8694 & 5.125 \\
\hline & 72 & 0.8694 & 5.125 \\
\hline Flat surface & Present study & 0.8694 & 5.125 \\
\hline & Kim and Wu [46] & $0.81 \pm 0.04$ & 4.5 \\
\hline
\end{tabular}

TABLE IV: Street speed $\left(V_{s t}\right)$ and street wavelength $(\lambda)$ at $R e=100$ for flow past a circular cylinder.

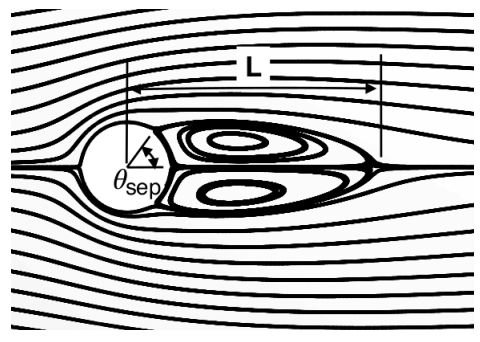

(a) $R e=40$

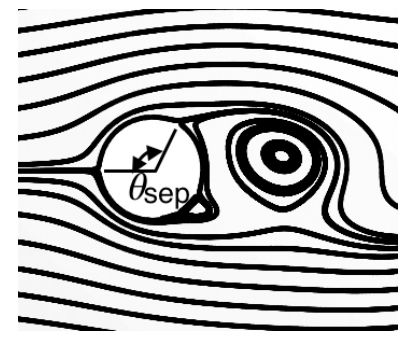

(b) $R e=100$, and 1000
FIG. 8: Schematics depicting definitions of the flow separation angle and the length of the stationary vortex pair.

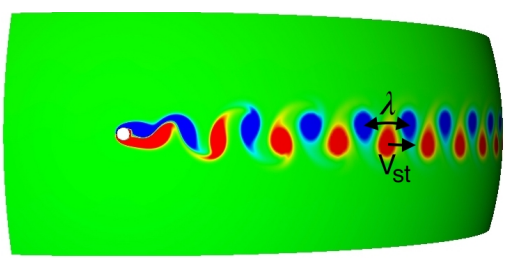

FIG. 9: Schematic showing notion of the street speed and street wavelength.

Three Reynolds numbers $\left(R e=\rho u_{\infty} L / \mu\right)$ of 40,100 and 500 are considered.

\section{Drag Coefficient, Lift Coefficient, and Strouhal Number}

The net force acting on the cylinder, due to pressure and viscous forces, is calculated using equation (2). The same definitions as in section III A 2 are used for $C_{d}, C_{l}$, and $S t$ with $L$ as the characteristic length instead of $D$.

The steady state values of $C_{d}$ at $R e=40$, in addition to the mean values at $R e=100$, and 500 , as well as RMS lift coefficient and Strouhal number at $R e=100$ and 500 are reported in table V. For $R e=100$, the mean drag coefficient for the flat embedding surface is 1.495 , which is in good agreement with that reported in the literature (e.g., 1.493 (Sharma and Eswaran [47])). For the spherical embedding surface, the mean drag coefficient is 1.494 for $R=72$, and is 1.477 for $R=12$. The change in $C_{d}$ with curvature is about $1 \%$ and the mean drag coefficient almost coincides with that for the flat embedding surface. For the cylindrical embedding surface, the mean drag coefficient is constant at 1.495 coinciding with that for the flat embedding surface. Similarly for $R e=40$ and 500 the maximum variation in the drag coefficient with the embedding surface radius is about $2.5 \%$, and $4.0 \%$, respectively, and $C_{d}$ values almost coincide with that for the flat embedding surface. Also, the RMS values of lift coefficient and the Strouhal number for the curved embedding surfaces, for both $R e=100$ and 500, almost coincide with that for the flat embedding surface.

\section{Length of Stationary Vortex Pair}

TableVI reports the lengths of the stationary vortex pair at $R e=40$. The lengths are in terms of geodesic distances for the curved embedding surfaces. The vortex pair length is 3.35 for the flat embedding surface, which agrees with that reported in Yoon et al. [49]. The maximum difference for the curved embedding surfaces is almost $1.5 \%$ relative to the flat embedding surface.

\section{Street Speed, and Street Wavelength}

Table VII reports street speed and wavelength for $R e=100$. The street speed is 0.875 for the flat embedding surface which agrees with the experimental value of $0.86 \pm 0.05$ reported in Kim and Wu [46]. The maximum difference for the curved embedding surfaces relative to the flat embedding surface is almost $0.11 \%$. The wavelength is 6.0 for the flat embedding surface, which almost agrees with the experimental value of 5.612 reported in $\mathrm{Kim}$ and $\mathrm{Wu}$ [46]. The maximum difference for 


\begin{tabular}{|c|c|c|c|c|c|c|c|c|}
\hline & & $R e=40$ & \multicolumn{3}{|c|}{$R e=100$} & \multicolumn{3}{|c|}{$R e=500$} \\
\hline Surface type & Radius of the surface & $C_{d}$ & $\begin{array}{c}C_{d} \\
\text { (mean) }\end{array}$ & $\begin{array}{c}C_{l} \\
(\mathrm{RMS})\end{array}$ & $S t$ & $\begin{array}{c}C_{d} \\
\text { (mean) }\end{array}$ & $\begin{array}{c}C_{l} \\
(\mathrm{RMS})\end{array}$ & $S t$ \\
\hline Spherical surface & 12 & 1.705 & 1.477 & 0.2 & 0.148 & 1.897 & 1.107 & 0.148 \\
\hline & 72 & 1.746 & 1.494 & 0.2 & 0.149 & 1.978 & 1.129 & 0.155 \\
\hline Cylindrical surface & 12 & 1.747 & 1.495 & 0.2 & $\overline{0.149}$ & 1.978 & 1.123 & 0.156 \\
\hline & 72 & 1.747 & 1.495 & 0.2 & 0.149 & 1.978 & 1.123 & 0.156 \\
\hline Flat surface & Present study & 1.747 & 1.495 & 0.2 & 0.149 & 1.977 & 1.121 & 0.156 \\
\hline & Sharma and Eswaran [47] & 1.767 & 1.493 & 0.192 & $\overline{0.149}$ & - & - & - \\
\hline & Sohankar et al. [48] & - & - & - & - & 1.89 & 1.13 & 0.174 \\
\hline
\end{tabular}

TABLE V: Mean drag coefficient, RMS lift coefficient, and Strouhal number for flow past a square cylinder cases.

\begin{tabular}{|c|c|c|}
\hline Surface type & Radius of the surface & L \\
\hline Spherical surface & 12 & 3.40 \\
\hline & 72 & 3.35 \\
\hline Cylindrical surface & 12 & 3.37 \\
\hline & 72 & 3.35 \\
\hline Flat surface & Present study & 3.35 \\
\hline & Yoon et al. [49] & 3.34 \\
\hline
\end{tabular}

TABLE VI: Length of the stationary vortex pair at $R e=40$ for the flow past a square cylinder cases.

the curved embedding surfaces is almost $2 \%$ relative to the flat embedding surface.

\section{Triangular Cylinder}

Flow past an equilateral triangle of edge length $(L)$ unity and oriented symmetrically with the vertical edge facing the downstream flow is considered. The Reynolds numbers $\left(R e=\rho u_{\infty} L / \mu\right)$ of 40,100 and 250 are considered.

Table VIII reports the values of drag coefficient, lift coefficient, and Strouhal number. For $R e=40$, the steady state value of the drag coefficient for the curved embedding surfaces differ by a maximum of about $2 \%$ relative to the flat embedding surface. For $R e=100$, the mean drag coefficient, RMS lift coefficient and Strouhal number for the curved embedding surfaces differ maximally by $2.7 \%, 5 \%$ and $2 \%$, respectively, relative to the flat embedding surface. For $R e=250$, relative to the flat embedding surface, the values of the mean drag coefficient, RMS lift coefficient and Strouhal number for the curved embedding surfaces differ by a maximum of about $3.4 \%, 3 \%$ and $2 \%$, respectively. Thus the value of these fluid dynamical quantities for the curved embedding surfaces almost coincide with that the for the flat embedding surface.

\section{The Curvature Term Contribution}

The aforementioned results indicate that the dynamics of the flow past a stationary circular, square, or triangular cylinder embedded on a curved surface is not significantly affected by the surface curvature, and is almost identical to that for a flat embedding surface. In other words, within the range of surface curvature values considered here, the dynamics of flow past a cylinder for each of the aforementioned cross-sections of the cylinder exhibits universality. To explore it further, a dimensional analysis was carried out yielding two non-dimensional parameters, i.e., the Reynolds number $\left(R e=\rho u_{\infty} L_{c} / \mu\right)$, and the ratio $R / L_{c}$. Here, $R$ is the radius of curvature of the surface, and $L_{c}$ is the characteristic length-scale, which is equal to the diameter of the cylinder (for the circular cylinder) or the edge length of the square (for square cylinder) or that of the triangle (for triangular cylinder). Moreover, the linear momentum equation in non-dimensional form (for the spherical embedding surface) is expressed,

$$
\begin{aligned}
& \frac{\partial \overrightarrow{u^{*}}}{\partial t^{*}}+\left(\overrightarrow{u^{*}} \cdot \nabla^{*}\right) \overrightarrow{u^{*}}+\left(\frac{1}{R e}\right) \Delta^{*} \overrightarrow{u^{*}} \\
& -2\left(\kappa L_{c}^{2}\right)\left(\frac{1}{R e}\right) \overrightarrow{u^{*}}+\nabla^{*} p^{*}=0,
\end{aligned}
$$

or for the spherical embedding surface, since the Gaussian curvature $\kappa=1 / R^{2}$,

$$
\begin{gathered}
\frac{\partial \overrightarrow{u^{*}}}{\partial t^{*}}+\left(\overrightarrow{u^{*}} \cdot \nabla^{*}\right) \overrightarrow{u^{*}}+\left(\frac{1}{R e}\right) \Delta^{*} \overrightarrow{u^{*}} \\
-2\left(\frac{1}{\left(R / L_{c}\right)^{2}}\right)\left(\frac{1}{R e}\right) \overrightarrow{u^{*}}+\nabla^{*} p^{*}=0,
\end{gathered}
$$

where the standard viscous term is $\left(\left(\frac{1}{R e}\right) \Delta^{*} \overrightarrow{u^{*}}\right)$ and an additional terms involving the curvature is $\left(-2\left(\frac{1}{\left(R / L_{c}\right)^{2}}\right)\left(\frac{1}{R e}\right) \overrightarrow{u^{*}}\right)$. The magnitudes of these two terms can be compared. For a given $R e$, the curvature term magnitude varies inversely with $\left(R / L_{c}\right)^{2}$. Thus, for the range of $R / L_{c}$ considered here, maximum relative magnitude of the curvature term corresponds to the 


\begin{tabular}{|c|c|c|c|}
\hline Surface type & Radius of the surface & $V_{s t} / u_{\infty}$ & $\lambda$ \\
\hline Spherical surface & 12 & 0.874 & 6.117 \\
\hline & 72 & 0.875 & 6.125 \\
\hline Cylindrical surface & 12 & 0.875 & 6.000 \\
\hline & 72 & 0.875 & 6.000 \\
\hline Flat surface & Present study & 0.875 & 6.000 \\
\hline & Kim and Wu [46] & $0.86 \pm 0.05$ & 5.612 \\
\hline
\end{tabular}

TABLE VII: Street speed $\left(V_{s t}\right)$ and street wavelength $(\lambda)$ at $R e=100$ for the flow past a square cylinder cases.

\begin{tabular}{|c|c|c|c|c|c|c|c|c|}
\hline & & $R e=40$ & \multicolumn{3}{|c|}{$R e=100$} & \multicolumn{3}{|c|}{$R e=250$} \\
\hline Surface type & Radius of the surface & $C_{d}$ & $\begin{array}{c}C_{d} \\
(\text { mean })\end{array}$ & $\begin{array}{c}C_{l} \\
(\mathrm{RMS})\end{array}$ & $S t$ & $\begin{array}{c}C_{d} \\
(\mathrm{mean})\end{array}$ & $\begin{array}{c}C_{l} \\
(\mathrm{RMS})\end{array}$ & $S t$ \\
\hline \hline Spherical surface & 12 & 1.503 & 1.770 & 0.305 & 0.200 & 2.025 & 0.462 & 0.196 \\
\hline & 72 & 1.535 & 1.818 & 0.321 & 0.204 & 2.098 & 0.476 & 0.200 \\
\hline Cylindrical surface & 12 & 1.535 & 1.819 & 0.321 & 0.204 & 2.097 & 0.476 & 0.200 \\
\hline & 72 & 1.536 & 1.819 & 0.321 & 0.204 & 2.097 & 0.476 & 0.200 \\
\hline Flat surface & Present study & 1.536 & 1.819 & 0.321 & 0.204 & 2.096 & 0.476 & 0.200 \\
\hline & Kumar De and Dalal [50] & 1.520 & 1.761 & 0.297 & 0.197 & 2.030 & 0.458 & 0.191 \\
\hline
\end{tabular}

TABLE VIII: Mean drag coefficient, RMS lift coefficient, and Strouhal number for flow past a triangular cylinder cases.

lower bound $R / L_{c}=12$, and the minimum relative magnitude corresponds to the upper bound $R / L_{c}=76$. On the other hand, $\kappa=0$ for the cylindrical and flat embedding surfaces, and hence the additional curvature term $-2\left(\kappa L_{c}^{2}\right)\left(\frac{1}{R e}\right) \overrightarrow{u^{*}}=0$ for these embedding surfaces.

In order to quantify the relative magnitude of Gaussian curvature term, we compare its magnitude with the viscous term magnitude. For the case of a circular cylinder embedded on a spherical surface, we find that the viscous term dominates in the region around the cylinder (see figure 10), and is at least four orders of magnitude larger than the curvature term, for the cases considered here. Therefore, for the simulations considered in the present study, the curvature term has an insignificant effect on the flow dynamics around the cylinder. For the cylindrical surface on the other hand, the Gaussian curvature, and therefore the curvature term, is identically equal to zero and does not affect the flow dynamics. In addition to the Gaussian curvature term, geometric constraint can affect the flow dynamics on a curved surface. Here, the constraint is that the intersection contour of the cylinder with the curved embedding surface has to confine itself to the curved surface. Therefore, there is a departure of the intersection contour from being a planar circle/square/triangle, whereas on a flat embedding surface it is a planar circle/square/triangle. For the case of a circular cylinder intersecting with a spherical embedding surface, the intersection is identical to a planar circle. The geometry in the region around the cylinder is therefore not significantly different from that for a flat surface, and does not considerably affect the flow dynamics. For an embedding cylindrical surface on the other hand, this departure is present. However, for the cases considered during the present study, the differences between the intersection contour and a plane circle remain relatively small. This can be quantified by evaluating the maximum variation of the z-coordinate along the intersection contour and also the change in the intersection contour length in comparison to a plane circle. The maximum variation of the $\mathrm{z}$-coordinate along the contour varies from 0.01040 for $R=12$ to 0.00174 for $R=72$. The percentage change in the contour length varies from $0.02419 \%$ for $R=12$ to $0.00318 \%$ for $R=72$. These values are evidently insignificant. The inconsiderable impact of the curvature term along with the trivial domain geometry changes, for the range of parameters considered in the present study, ultimately resulted in the observed similarity in the flow past a cylinder dynamics on curved surfaces, in comparison to that on a flat surface. Similarly for the square and triangular cylinders, the effect of the two aforementioned parameters on the flow dynamics around the cylinder is insignificant. Consequently, the effect of the embedding surface curvature on the flow dynamics around the square and triangular cylinder is also insignificant, for the range of parameters considered in this study.

\section{CONCLUSION}

The present study investigated the dynamics of the flows past circular, square and triangular cylinders embedded on spherical and cylindrical surfaces. Our main objective was to explore the embedding surface curvature effects on the flow dynamics. Moreover, the present study may be considered a precursor for potential future soapfilm experiments on such curved configurations. For the range of parameters considered here, no significant differences were observed in the flow dynamics on the curved surfaces, when compared with the flow on a flat surface. 


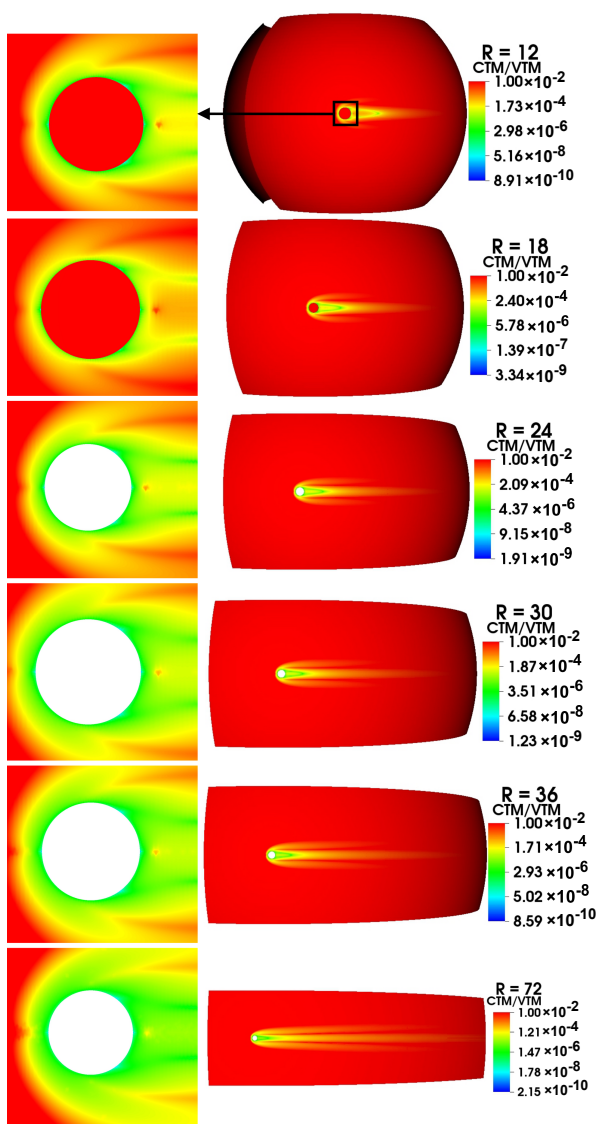

(a) $R e=40$
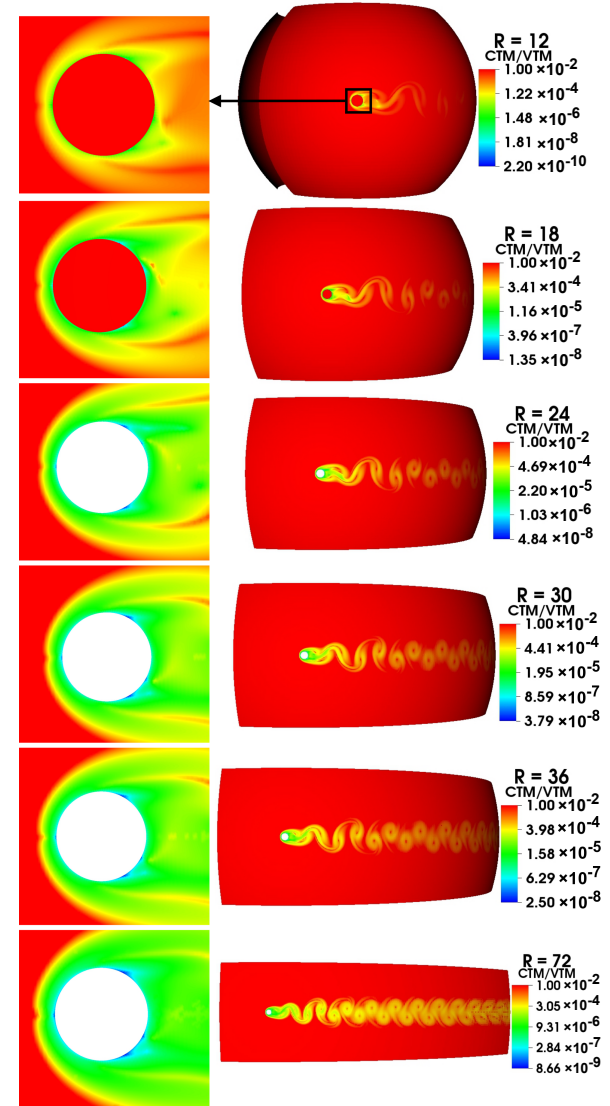

(b) $R e=100$
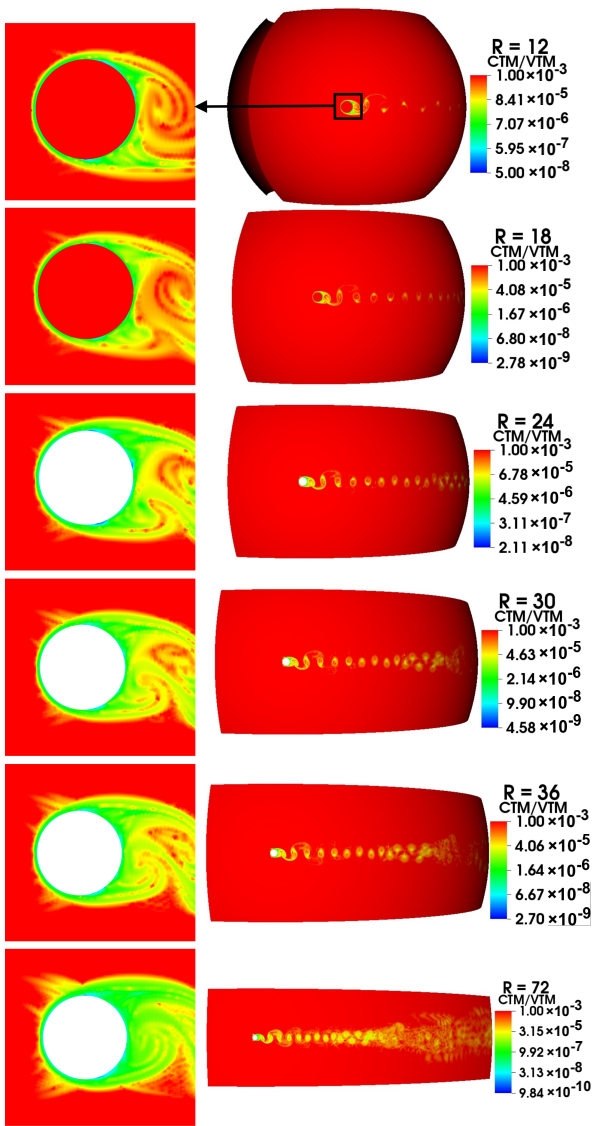

(c) $R e=1000$

FIG. 10: Ratio between the curvature term magnitude (CTM) and viscous term magnitude(VTM) for the circular cylinder test cases. 
Accordingly, the key conclusion is that the dynamics of a flow past a stationary circular, square or triangular cylinder embedded on a surface are not significantly affected by the curvature of the embedding surface for the range of parameters considered here. We provided a quantitative justification for such observations, addressing both the magnitude of the curvature term in the momentum equation (relative to the viscous term) and the domain geometry changes due to the embedding surface curvature. Both factors were found to be insignificant for all test cases considered here, resulting in the observed surface curvature insensitivity. While previous vortex dynamics investigations reported a significant impact of the surface curvature on the flow dynamics, this paper points out to a different type of flow that is insensitive to the embedding surface curvature. This paves the way to a more thorough discussion on what makes a surface flow sensitive to the embedding surface curvature since previous investigations reported a measurable effect due to curvature for some flow types while our work noted the absence of a significant curvature effect for the flow past a cylinder. We conjecture that the dynamics of the flow past bluff bodies is essentially universal. While our investigation point to this universality based on sampling of the non-dimensional curvature in the range $0 \leq \kappa D \leq 1 / 12$, it is imperative that even higher curvatures, than the ones considered here, be explored to more convincingly settle this issue of universality.

\section{Appendix A: Primer on Discrete Exterior Calculus (DEC)}

Here, we present some of the essential ideas of exterior calculus for the uninitiated reader with the cautionary note that this section is not meant to be an exhaustive introduction to this subject. Exterior calculus deals with the calculus of differential forms. While scalars, vectors and tensors are the fundamental quantities of interest in vector calculus, differential forms ( $k$-forms) are the key quantities in exterior calculus. A good description of differential forms, from the point of view of their applications to the science and engineering, is available in [51], and is largely referred to in the ensuing discussion. A differential form can be thought of as "the things which occur under integral signs" [52]. For example, given a line integral of a vector $\mathbf{v}, \int \mathbf{v} \cdot \mathbf{d l}, \mathbf{v} \cdot \mathbf{d} \mathbf{l}$ represents a 1 -form $v^{1}$. Similarly, a 2-form $q^{2}=\mathbf{q} \cdot \mathbf{d A}$ appears in surface integrals, a 3 -form $s^{3}=s d V$ appears in volume integrals, and a 0 -form $p^{0}$ is a scalar function since a 0 -integral is trivial. Here, the superscript on the form represents the dimension of the form. The discrete forms, on the other hand, are the entire integral quantities. For example, integration of a vector $\mathbf{v}$ over a mesh edge $\int \mathbf{v} \cdot \mathbf{d l}$ is a discrete 1 -form $v^{1}$. Similarly, a discrete 2 -form $q^{2}$ is the surface integral $\int \mathbf{q} \cdot \mathbf{d A}$ over a mesh face, a discrete 3form is the volume integral $s^{3}=\int s d V$ on a cell volume, and a discrete 0 -form is just a scalar field value at a mesh vertex/node since the integration over a point is trivial and it just returns the value of the integrand at that point. The interpretation of discrete 0 -form and that of the discrete 1-form remain essentially the same whether it is two-dimensional space (2D) or three-dimensional space (3D). However, the discrete 2 -form is a cell average integral of a scalar field in 2D rather than integral of a vector field on a mesh face as in $3 \mathrm{D}$, since the mesh cells are represented by areas in 2D. A 3 -form does not exist in 2D, and the 2-form essentially behaves like the 3 -form does in 3D. Other key concepts include the exterior derivative (d) which implies multidimensional differentiation, and is a generalization of the usual curl, divergence and gradient operations of calculus; the Hodge star operator (*) which has a metric term incorporated in it; the $e x$ terior product or wedge product $(\wedge)$, which implies multiplication of differential forms. The exterior derivative operator when applied to a form, takes it to the next higher dimensional form. In 3D, the gradient of a scalar, $\nabla p=\mathbf{q}$ in vector calculus is equivalent to $\mathrm{d} p^{0}=q^{1}$ in exterior calculus. Here, the resulting vector $\mathbf{q}$ is best represented as a 1 -form $q^{1}$. The curl of a vector, $\nabla \times \mathbf{v}=\mathbf{a}$ is equivalent to $\mathrm{d} v^{1}=a^{2}$, and the resulting vector a (in vector calculus) is represented as a 2-form (in exterior calculus). An equivalent operation to the divergence of a vector, i.e., $\nabla \cdot \mathbf{u}=b$ is $\mathrm{d} u^{2}=b^{3}$, resulting into a 3 -form which is a scalar. In $2 \mathrm{D}$, only two primary differentiation operations, i.e., gradient $\nabla$, and $2 \mathrm{D}$ curl, $\nabla \times_{2 D}$, should be used and the divergence does not add any useful content. The gradient of a scalar is equivalent to the exterior derivative operating on a 0 -form, looks the same as in 3D, and implies the difference between two node values / discrete 0-forms. The curl on edges lying in a plane calculates the vector component normal to the plane. The result of a $2 \mathrm{D} \operatorname{curl} \nabla \times_{2 D} \mathbf{v}=\frac{\partial v_{y}}{\partial x_{x}}-\frac{\partial v_{x}}{\partial x_{y}}$ is a scalar, which is equivalent to a 2 -form resulting from the application of an exterior derivative to a 1-form (on an edge). The Hodge star operates on a form in one dimension and the resultant form is in the reflected dimension, i.e., $* a^{k}=b^{N-k}, N$ standing for the space dimension. The Hodge star operates on a $k$-form $a^{k}$ and results in to a $(N-k)$-form. In 3D, Hodge star operating on a 0 -form (scalar point value) results in to a 3 -form (cell average of scalar) and vice-versa. The Hodge star applied to a 1 -form (vector component along a line) results in a 2 -form (vector component normal to a face) and viceversa. In practice, this means that a discrete Hodge star operator transfers the values on a mesh to a dual mesh (as well as changes their dimension). Since every well formed mesh has an infinite number of well formed dual meshes, one need to identify which dual mesh is being used for the definition of the discrete Hodge star matrices. The voronoi dual mesh (circumcentric dual mesh), or the median dual mesh (barycentric dual mesh) are the examples of dual meshes for unstructured (triangular or tetrahedral) primary meshes. The discrete Hodge star $\left(*_{0}\right)$ operating on cell node (vertex) values (0-forms) of a tetrahedral mesh results in cell average values (dual 3- 
forms) for the Voronoi cells that surround those nodes. Similarly the inverse operator $\left(*_{1}^{-1}\right)$ operating on 1-forms lying on the line between circumcenters of two adjacent triangles (an edge of the dual mesh) results in normal flux values on the cell faces (2-forms on the primal mesh). In $2 \mathrm{D}$, a discrete $*_{0}$ operation transfers mesh vertex values (primal 0-forms) to Voronoi cell values (dual 2-forms), and $*_{0}^{-1}$ transfers the cell average values (dual 2-forms) to mesh vertex values (primal 0 -forms). The discrete $*_{1}$ operator transfers the 1 -forms (primal edge values) to 1 -forms (dual edge values). The wedge product of two forms implies multiplication of these forms. The dimension of the resultant form of the wedge product is the sum of the dimensions of the two forms in the wedge product, i.e., $p^{j} \wedge q^{k}=r^{j+k}$ for $j+k \leq N$, with a property $p^{j} \wedge q^{k}=(-1)^{j k} q^{k} \wedge p^{j}$. The wedge product is also associative, i.e., $\left(p^{j} \wedge q^{k}\right) \wedge r^{l}=p^{j} \wedge\left(q^{k} \wedge r^{l}\right)$ for $j+k+l \leq N$. In $3 \mathrm{D}$, the wedge product is either similar to a regular multiply, a dot-product, or a cross-product depending on the dimension of the two forms involved in the wedge product. If either operand is a scalar 0 -form, the wedge product implies a regular multiplication of the operands. The wedge product implies a dot product of the operands only when one of the operands is a 1-form and the other is a 2-form. The wedge product of two 1forms implies cross product on the vector proxies of these 1 -forms (the operands) which results in a 2 -form. In 2D, the wedge product is either a regular multiplication or a $2 \mathrm{D}$ cross product. Similar to 3D, if either operand is a 0 -form, a regular multiply is called for. For the two forms $p^{1}$ and $q^{1}$ associated with the vectors $\mathbf{p}$ and $\mathbf{q}$, respectively, the wedge product $p^{1} \wedge q^{1}$ is equivalent to $\mathbf{p} \times_{2 D} \mathbf{q}=p_{x} q_{y}-p_{y} q_{x}$.

Using integral quantities / discrete forms as the primary unknowns for a numerical method allows one to transform any continuous PDE system in to a discrete algebraic system such that all errors/approximations occur at the algebraic level only and not in the approximation of the differential operators of the PDE [53]. Thus, DEC can mimic physical and mathematical properties of the continuous PDE system. Moreover, DEC is coordinate independent, making it a more convenient numerical tool to investigate flows over arbitrary curved surfaces/manifolds. As already mentioned above, the implementation of DEC operators requires a primal simplicial complex (triangular or tetrahedral mesh in two or three dimensions, respectively), along with a dual mesh associated to it (usually circumcentric). In two dimensions, the primal simplicial complex is the set of nodes, edges, and triangles. The dual mesh consists of dual polygons, edges and nodes associated with the primal mesh objects. When discretizing a physical problem using DEC, the physical fields are discretely expressed as scalars representing the integration of these $k$-forms on $k$-dimensional primal/dual mesh objects as already mentioned above. Some of the representative references in DEC include [33, 51, 52, 54-62].

\section{Appendix B: Numerical Procedure}

The numerical scheme developed by Mohamed et al. [33] is briefly discussed here along with any modifications adopted during the current implementation. Unlike the stream function formulation employed in Mohamed et al. [33], the present simulations adopt the primitive variable formulation. Moreover, the governing equations include the Gaussian curvature term to account for the embedding surface curvature [63].

The governing equations consist of the incompressible Navier-Stokes equations, i.e., linear momentum equations and the continuity equation or incompressibility constraint. A coordinate invariant form of the linear momentum equation, assuming constant properties and unit density, reads [33]

$$
\begin{gathered}
\frac{\partial \mathbf{u}^{b}}{\partial t}+\mu(\delta \mathrm{d}+\mathrm{d} \delta) \mathbf{u}^{b}-2 \mu \kappa \mathbf{u}^{b}+\mathcal{L}_{\mathbf{u}} \mathbf{u}^{b} \\
-\frac{1}{2} \mathrm{~d}\left(\mathbf{u}^{\mathrm{b}}(\mathbf{u})\right)+\mathrm{d} p=0 .
\end{gathered}
$$

Here, $\mathbf{u}^{b}, \kappa, \mu, \mathrm{d}, \delta, \mathcal{L}_{\mathbf{u}}$, and $p$ denote the velocity 1 -form corresponding to the velocity vector $\mathbf{u}$, Gaussian curvature, dynamic viscosity, exterior derivative, codifferential operator, Lie derivative, and pressure 0-form, respectively. The Gaussian curvature term $\left(2 \mu \kappa \mathbf{u}^{b}\right)$ results from the divergence of the deformation tensor and the non-commutativity of the second covariant derivative in curved spaces [63]. The codifferential operator is defined as $\delta=(-1)^{N(k-1)+1} * \mathrm{~d} *$, which operates on a $k$-form and results in a $(k-1)$-form, $N$ being the space dimension, and $*$ denotes for the Hodge star. The incompressibility condition reads

$$
\delta \mathbf{u}^{b}=0 .
$$

The term $\mu(\delta \mathrm{d}+\mathrm{d} \delta) \mathbf{u}^{b}$ is the diffusion term. The operator $(\delta \mathrm{d}+\mathrm{d} \delta)$ is the Laplace-deRham operator. The term $\mathcal{L}_{\mathbf{u}} \mathbf{u}^{b}-\frac{1}{2} \mathrm{~d}\left(\mathbf{u}^{b}(\mathbf{u})\right)$ is the advection term (it is the expression of covariant derivative), with the Lie derivative term $\left(\mathcal{L}_{\mathbf{u}} \mathbf{u}^{b}\right)$ evaluating the change of the velocity 1 -form $\mathbf{u}^{b}$ along the velocity vector field $\mathbf{u}$, and the term $\mathbf{u}^{\mathrm{b}}(\mathbf{u})$ representing the dot product of the vector field $\mathbf{u}$ with itself. The Lie derivative term is expressed as [33]

$$
\mathcal{L}_{\mathbf{u}} \mathbf{u}^{\mathrm{b}}=\mathrm{d}\left(\mathbf{u}^{\mathrm{b}}(\mathbf{u})\right)+i_{\mathbf{u}} \mathrm{d} \mathbf{u}^{\mathrm{b}},
$$

where $i_{x} \alpha$ is the interior product of a $k$-form $\alpha$ with a vector field $\mathbf{x}$, which can be expressed as [33]

$$
i_{\mathbf{x}} \alpha=(-1)^{k(N-k)} *\left(* \alpha \wedge \mathbf{x}^{b}\right),
$$

where $\wedge$ stands for the wedge product, and $x^{b}$ is a form which corresponds to the vector field $\mathbf{x}$. Accordingly, considering the incompressibility condition, equation (B1) can be expressed as 


$$
\begin{gathered}
\frac{\partial \mathbf{u}^{b}}{\partial t}+\mu \delta \mathrm{d} \mathbf{u}^{b}-2 \mu \kappa \mathbf{u}^{b}+i_{\mathbf{u}} \mathrm{d} \mathbf{u}^{b} \\
+\frac{1}{2} \mathrm{~d}\left(\mathbf{u}^{b}(\mathbf{u})\right)+\mathrm{d} p=0
\end{gathered}
$$

The dynamic pressure 0 -form is defined as $p^{d}=p+$ $\frac{1}{2}\left(\mathbf{u}^{b}(\mathbf{u})\right)$, and substituting with the definition of $\delta=$ $(-1)^{N+1} * \mathrm{~d} *$ (since $\delta$ in equation (B5) operates on the 2 -form d $\mathbf{u}^{b}$, hence $k=2$ ), equation (B5) then becomes

$$
\frac{\partial \mathbf{u}^{\mathrm{b}}}{\partial t}+(-1)^{N+1} \mu * \mathrm{~d} * \mathrm{~d} \mathbf{u}^{\mathrm{b}}-2 \mu \kappa \mathbf{u}^{\mathrm{b}}+i_{\mathbf{u}} \mathrm{d} \mathbf{u}^{\mathrm{b}}+\mathrm{d} p^{d}=0 .
$$

Substituting for the interior product term with equation (B4), equation (B6) reads

$$
\begin{aligned}
& \frac{\partial \mathbf{u}^{\mathrm{b}}}{\partial t}+(-1)^{N+1} \mu * \mathrm{~d} * \mathrm{~d} \mathbf{u}^{\mathrm{b}}-2 \mu \kappa \mathbf{u}^{\mathrm{b}} \\
& +(-1)^{N-2} *\left(\mathbf{u}^{\mathrm{b}} \wedge * \mathrm{~d} \mathbf{u}^{\mathrm{b}}\right)+\mathrm{d} p^{d}=0,
\end{aligned}
$$

where the order of the wedge product was flipped using the relation $\alpha \wedge \beta=(-1)^{k l} \beta \wedge \alpha$, with $\alpha=*$ d $\mathbf{u}^{b}$ being an $(N-2)$-form and $\beta=\mathbf{u}^{b}$ being a 1 -form.

As mentioned earlier (see appendix A), DEC discretizations in $2 \mathrm{D}$ require a primal triangular mesh along with a dual mesh, which in our implementation is chosen to be the circumcentric dual. The dual to a primal triangle is its circumcenter, the dual to a primal edge is the perpendicular dual edge connecting the circumcenters of the two triangles sharing this primal edge, and the dual to a primal node is the polygon formed by the edges dual to the primal edges connected to this node. The positive orientation of both primal mesh (triangles / primal 2-simplices) and dual mesh (polygons / dual 2-simplices) is assumed to be counterclockwise. The orientation of the primal edges (primal 1-simplices) is arbitrary, however their orientations induce the dual edges orientations. The dual edges are oriented simply by rotating the primal edge orientation 90 degrees along the triangles orientation (i.e., counterclockwise).

The discretization in two-dimensional space is considered. The DEC discretization of the aforementioned governing equations requires first to specify where the discrete variables are defined on the mesh objects. The smooth forms are then replaced by their discrete counterparts, and the smooth exterior calculus operators are substituted with the appropriate discrete operators. For the current discretization, the velocity 1 -form $\mathbf{u}^{b}$ in all terms (except one to be discussed later) is discretized as a dual 1-form, representing the velocity integration along the dual edges. This choice results in requiring the dynamic pressure $p^{d}$ to be defined as a dual 0-form, i.e, at the triangles circumcenters so that the term $\mathrm{d} p^{d}$ is a dual 1-form, consistent with the choice of $\mathbf{u}^{b}$ as the dual 1form. Since the convective term $*\left(\mathbf{u}^{b} \wedge * \mathrm{~d} \mathbf{u}^{b}\right)$ is defined on the dual edge, $\left(\mathbf{u}^{b} \wedge * \mathrm{~d} \mathbf{u}^{b}\right)$ is defined on the primal edges. As $\mathbf{u}^{b}$ is a 1 -form, and hence $* \mathrm{~d} \mathbf{u}^{b}$ is a 0 -form (in
2D), and therefore the operands of the wedge product are a 1-form and a 0 -form, such that the resultant (primal 1 -form) must be defined on the primal edges. This implies that both wedge product operands are defined on primal mesh objects. Accordingly, the velocity 1-form in the first operand $\left(\mathbf{u}^{b}\right)$ of the wedge product term is defined on the primal edges, whereas the velocity 1-form in the second operand $\left(* \mathrm{~d} \mathbf{u}^{\mathrm{b}}\right)$ of the wedge product term is defined on the dual edges, making the 0 -form $* \mathrm{~d} \mathbf{u}^{b}$ to be defined on the primal nodes. Such definitions of discrete forms determine the corresponding definitions for the discrete $\mathrm{d}, *$ and $\wedge$ operators. As discussed in [33], the discrete exterior derivative operator $\mathrm{d}_{k}$ operates on primal $k$-forms and maps them to primal $(k+1)$-forms. The discrete exterior derivative operator that operates on dual $k$-forms and maps them to dual $(k+1)$-forms is the transpose of the $\mathrm{d}_{(N-K-1)}$ operator, i.e., $\mathrm{d}_{(N-K-1)}^{T}$, with a negative sign only for the $\mathrm{d}_{0}^{T}$ operator in $2 \mathrm{D}$ (due to the defined mesh orientation convention). The discrete exterior derivative operator $\mathrm{d}_{k}$ is expressed as a sparse matrix that is defined as the transpose of the boundary operator for the $(k+1)$-simplices. The discrete Hodge star operator $*_{k}$ operates on primal $k$-forms and maps them to dual $(N-k)$-forms. The inverse map of the discrete $*_{k}$ operator is $*_{k}^{-1}$, which operates on dual $(N-k)$-forms and maps them to primal $k$-forms. The discrete Hodge star operator $*_{k}$ is expressed as a diagonal matrix with the $i$-th diagonal element being the ratio of the volume of the dual $(N-k)$-cell $\star \sigma_{i}^{k}$ to the volume of its primal $k$-simplex $\sigma_{i}^{k}$, i.e. $\frac{\star \sigma_{i}^{k}}{\sigma_{i}^{k}}$. The discrete definition of the wedge product is discussed in the following paragraph.

The DEC discretization of equation (B7) in $2 \mathrm{D}$ then reads

$$
\begin{gathered}
\frac{U^{n+1}-U^{n}}{\Delta t}-\mu *_{1} \mathrm{~d}_{0} *_{0}^{-1}\left[\left[-\mathrm{d}_{0}^{T}\right] U+\mathrm{d}_{b} V\right]-2 \mu \kappa U \\
+*_{1} W_{V} *_{0}^{-1}\left[\left[-\mathrm{d}_{0}^{T}\right] U+\mathrm{d}_{b} V\right]+\mathrm{d}_{1}^{T} P^{d}=0 .
\end{gathered}
$$

$U, V$, and $P^{d}$ are the vectors containing the discrete dual velocity 1 -forms for all mesh dual edges, the primal velocity 1-forms for all mesh primal edges, and the dynamic pressure dual 0-forms for all mesh dual vertices, respectively. The discrete time interval is expressed as $\Delta t$. Note that the time-centering of all terms except the time derivative one is discussed later in this section. The $W_{V}$ matrix represents the discrete wedge product of the primal velocity 1 -form with the 0 -form $(* \mathrm{~d} u)$, and contains the values of the primal velocity 1 -form. The boundary of the 2-cells (polygons), dual to the primal nodes lying on the domain boundary, includes primal boundary edges. Accordingly, the $\left[-\mathrm{d}_{0}^{T}\right]$ matrix, expressed as the transpose boundary operator of these dual 2-cells, is complemented by an additional operator $\mathrm{d}_{b}$ accounting for the primal boundary edges. The operation $\left[-\mathrm{d}_{0}^{T}\right] U$ evaluates the circulation of the velocity forms $u$ along the 
dual 2-cells boundaries. The operation $\mathrm{d}_{b} V$ complements the velocity circulation for the dual 2-cells part of whose boundaries consist of primal edges.

Let $U^{*}$ be a vector containing mass flux primal 1-form $u^{*}$ for all mesh primal edges. We then have $U^{*}=-*_{1}^{-1} U$ and $U=*_{1} U^{*}$. After this substitution, equation (B8) can be rewritten as

$$
\begin{gathered}
*_{1}\left[\frac{\left(U^{*}\right)^{n+1}-\left(U^{*}\right)^{n}}{\Delta t}\right]-\mu *_{1} \mathrm{~d}_{0} *_{0}^{-1}\left[\left[-\mathrm{d}_{0}^{T}\right] *_{1} U^{*}+\mathrm{d}_{b} V\right] \\
-2 \mu \kappa *_{1} U^{*}+*_{1} W_{V} *_{0}^{-1}\left[\left[-\mathrm{d}_{0}^{T}\right] *_{1} U^{*}+\mathrm{d}_{b} V\right] \\
+\mathrm{d}_{1}^{T} P^{d}=0 .
\end{gathered}
$$

Applying the Hodge star operator $*_{1}^{-1}$ to equation (B9), and considering the property $*_{1}^{-1} *_{1}=-1$, we have

$$
\begin{gathered}
-\frac{\left(U^{*}\right)^{n+1}-\left(U^{*}\right)^{n}}{\Delta t}+\mu \mathrm{d}_{0} *_{0}^{-1}\left[\left[-\mathrm{d}_{0}^{T}\right] *_{1} U^{*}+\mathrm{d}_{b} V\right] \\
+2 \mu \kappa U^{*}-W_{V} *_{0}^{-1}\left[\left[-\mathrm{d}_{0}^{T}\right] *_{1} U^{*}+\mathrm{d}_{b} V\right] \\
+*_{1}^{-1} \mathrm{~d}_{1}^{T} P^{d}=0 .
\end{gathered}
$$

Treating the viscous and curvature terms implicitly, and the convection term explicitly, we can write

$$
\begin{gathered}
{\left[-\frac{1}{\Delta t} I+\mu \mathrm{d}_{0} *_{0}^{-1}\left[-\mathrm{d}_{0}^{T}\right] *_{1}+2 \mu \kappa I\right]\left(U^{*}\right)^{n+1}} \\
+*_{1}^{-1} \mathrm{~d}_{1}^{T}\left(P^{d}\right)^{n+1}=F
\end{gathered}
$$

with

$$
\begin{gathered}
F=\frac{-1}{\Delta t}\left(U^{*}\right)^{n}-\mu \mathrm{d}_{0} *_{0}^{-1} \mathrm{~d}_{b} V^{n} \\
+W_{V}^{n} *_{0}^{-1}\left[\left[-\mathrm{d}_{0}^{T}\right] *_{1}\left(U^{*}\right)^{n}+\mathrm{d}_{b} V^{n}\right] .
\end{gathered}
$$

The continuity equation is expressed as

$$
\left[\mathrm{d}_{1}\right]\left(U^{*}\right)^{n+1}+[0]\left(P^{d}\right)^{n+1}=0 .
$$

Equations (B11) - (B13) form a linear system of equations with $U^{*}$ and $P^{d}$ at time $(n+1)$ to be the mass flux and dynamic pressure unknown degrees of freedom. This linear system is solved along with the boundary conditions depicted in figure 1.
[1] Stefanella Boatto and Jair Koiller, "Vortices on closed surfaces," in Geometry, Mechanics, and Dynamics (Springer, 2015) pp. 185-237.

[2] Dan Hu, Pingwen Zhang, and E Weinan, "Continuum theory of a moving membrane," Physical Review E 75, 041605 (2007).

[3] David R Nelson, "Toward a tetravalent chemistry of colloids," Nano Letters 2, 1125-1129 (2002).

[4] Peng Song, Dan Hu, and Pingwen Zhang, "Numerical simulation of fluid membranes in two-dimensional space," Communications in Computational Physics 3, 794-821 (2008).

[5] Mark J Bowick and Luca Giomi, "Two-dimensional matter: order, curvature and defects," Advances in Physics 58, 449-563 (2009).

[6] C Köhler, R Backofen, and A Voigt, "Relaxation of curvature-induced elastic stress by the asaro-tillergrinfeld instability," EPL (Europhysics Letters) 111, 48006 (2015).

[7] Jon Karl Sigurdsson and Paul J Atzberger, "Hydrodynamic coupling of particle inclusions embedded in curved lipid bilayer membranes," Soft matter 12, 6685-6707 (2016).

[8] Sebastian Reuther and Axel Voigt, "Solving the incompressible surface navier-stokes equation by surface finite elements," Physics of Fluids 30, 012107 (2018).

[9] Michael Nestler, Ingo Nitschke, Simon Praetorius, and Axel Voigt, "Orientational order on surfaces: The coupling of topology, geometry, and dynamics," Journal of Nonlinear Science 28, 147-191 (2018).

[10] Ingo Nitschke, Sebastian Reuther, and Axel Voigt, "Hydrodynamic interactions in polar liquid crystals on evolving surfaces," Physical Review Fluids 4, 044002 (2019).

[11] Ari M Turner, Vincenzo Vitelli, and David R Nelson, "Vortices on curved surfaces," Reviews of Modern Physics 82, 1301 (2010).
[12] Sebastian Reuther and Axel Voigt, "The interplay of curvature and vortices in flow on curved surfaces," Multiscale Modeling \& Simulation 13, 632-643 (2015).

[13] Takashi Sakajo and Yuuki Shimizu, "Point vortex interactions on a toroidal surface," Proceedings of the Royal Society A: Mathematical, Physical and Engineering Sciences 472, 20160271 (2016).

[14] Nicholas Syred, A Khalatov, A Kozlov, A Shchukin, and R Agachev, "Effect of surface curvature on heat transfer and hydrodynamics within a single hemispherical dimple," J. Turbomach. 123, 609-613 (2000).

[15] XiLin Xie, Yu Chen, and Qian Shi, "Some studies on mechanics of continuous mediums viewed as differential manifolds," Science China Physics, Mechanics and Astronomy 56, 432-456 (2013).

[16] Qian Shi, Yu Chen, and Xilin Xie, "Interplay of surface geometry and vorticity dynamics in incompressible flows on curved surfaces," Applied Mathematics and Mechanics 38, 1191-1212 (2017).

[17] T. Von Kármán, "Uber den mechanismus des flussigkeitsund luftwiderstandes," Phys. Z. , 49-59 (1912).

[18] G. J. Taylor, "Pressure distribution on a cylinder," Techn. Rep. of Adv. Comm. for Aeron., London 1916, 32 (1915).

[19] C. Wieselsberger, "Neuere feststellungen uber die gesetze des flussigkeits und luftwiderstands," Phys. Z. 22, 321 (1921).

[20] A. Fage, "The effects of turbulence and surface roughness on the drag of a circular cylinder," Rep, Memo. 1283, 1 (1929).

[21] A. Thom, "The flow past circular cylinders at low speeds," Proceedings of the Royal Society of London. Series A, Containing Papers of a Mathematical and Physical Character 141, 651-669 (1933).

[22] H. Niemann and N. Hölscher, "A review of recent experiments on the flow past circular cylinders," Journal 
of Wind Engineering and Industrial Aerodynamics 33 197-209 (1990).

[23] Xiaodong Wu, Fei Ge, and Youshi Hong, "A review of recent studies on vortex-induced vibrations of long slender cylinders," Journal of Fluids and Structures 28, 292-308 (2012).

[24] A. Rao, A. Radi, J. S. Leontini, M. C. Thompson, J. Sheridan, and K. Hourigan, "A review of rotating cylinder wake transitions," Journal of Fluids and Structures 53, 2-14 (2015).

[25] Yu Zhou and Md Mahbub Alam, "Wake of two interacting circular cylinders: a review," International Journal of Heat and Fluid Flow 62, 510-537 (2016).

[26] Rajesh Kumar and NK Singh, "Review of flows past arrays of elliptic and square cylinders," in Advances in Fluid and Thermal Engineering (Springer, 2019) pp. 837847.

[27] Morteza Gharib and Philip Derango, "A liquid film (soap film) tunnel to study two-dimensional laminar and turbulent shear flows," Physica D: Nonlinear Phenomena 37, 406-416 (1989).

[28] C-Y Wen, C-L Yeh, M-J Wang, and C-Y Lin, "On the drag of two-dimensional flow about a circular cylinder," Physics of Fluids 16, 3828-3831 (2004).

[29] Lai-Bing Jia and Xie-Zhen Yin, "Response modes of a flexible filament in the wake of a cylinder in a flowing soap film," Physics of Fluids 21, 101704 (2009).

[30] C-Y Wen and C-Y Lin, "Two-dimensional vortex shedding of a circular cylinder," Physics of Fluids 13, 557-560 (2001).

[31] MA Rutgers, XL Wu, and WB Daniel, "Conducting fluid dynamics experiments with vertically falling soap films," Review of Scientific Instruments 72, 3025-3037 (2001).

[32] Peter Vorobieff and Robert E Ecke, "Cylinder wakes in flowing soap films," Physical review E 60, 2953 (1999).

[33] M. S. Mohamed, A. N. Hirani, and R. Samtaney, "Discrete exterior calculus discretization of incompressible navier-stokes equations over surface simplicial meshes," Journal of Computational Physics 312, 175-191 (2016).

[34] J. M. Shi, D. Gerlach, M. Breuer, G. Biswas, and F. Durst, "Heating effect on steady and unsteady horizontal laminar flow of air past a circular cylinder," Physics of Fluids 16, 4331-4345 (2004).

[35] D. Russell and Z. J. Wang, "A cartesian grid method for modeling multiple moving objects in $2 \mathrm{~d}$ incompressible viscous flow," Journal of Computational Physics 191, 177-205 (2003).

[36] D. Le, B. C. Khoo, and J. Peraire, "An immersed interface method for viscous incompressible flows involving rigid and flexible boundaries," Journal of Computational Physics 220, 109-138 (2006).

[37] E. Stålberg, A. Brüger, P. Lötstedt, A. V. Johansson, and D. S. Henningson, "High order accurate solution of flow past a circular cylinder," Journal of Scientific Computing 27, 431-441 (2006).

[38] Mitutosi Kawaguti, "Numerical solution of the navierstokes equations for the flow around a circular cylinder at reynolds number 40," Journal of the Physical Society of Japan 8, 747-757 (1953).

[39] S. C. R. Dennis and Gau-Zu Chang, "Numerical solutions for steady flow past a circular cylinder at Reynolds numbers up to 100," Journal of Fluid Mechanics 42, 471-489 (1970).

[40] Ronald D Henderson and George Em Karniadakis, "Un- structured spectral element methods for simulation of turbulent flows," Journal of Computational Physics 122, 191-217 (1995).

[41] GJ Sheard, K Hourigan, and MC Thompson, "Computations of the drag coefficients for low-reynolds-number flow past rings," Journal of Fluid Mechanics 526, 257275 (2005).

[42] BN Rajani, Harsha Gopal Lanka, and Sekhar Majumdar, "Laminar flow past a circular cylinder at reynolds number varying from 50 to 5000," (2005).

[43] Mitutosi Kawaguti and Padam Jain, "Numerical study of a viscous fluid flow past a circular cylinder," Journal of the Physical Society of Japan 21, 2055-2062 (1966).

[44] Sadatoshi Taneda, "Experimental investigation of the wakes behind cylinders and plates at low reynolds numbers," Journal of the Physical Society of Japan 11, 302307 (1956).

[45] CJ Apelt, The steady flow of a viscous fluid past a circular cylinder at Reynolds numbers 40 and 44 (HM Stationery Office, 1961).

[46] Ildoo Kim and XL Wu, "Unified strouhal-reynolds number relationship for laminar vortex streets generated by different-shaped obstacles," Physical Review E 92, 043011 (2015).

[47] Atul Sharma and V Eswaran, "Heat and fluid flow across a square cylinder in the two-dimensional laminar flow regime," Numerical Heat Transfer, Part A: Applications 45, 247-269 (2004).

[48] Ahmad Sohankar, C Norberg, and L Davidson, "Simulation of three-dimensional flow around a square cylinder at moderate reynolds numbers," Physics of fluids 11, 288-306 (1999).

[49] Dong-Hyeog Yoon, Kyung-Soo Yang, and Choon-Bum Choi, "Flow past a square cylinder with an angle of incidence," Physics of fluids 22, 043603 (2010).

[50] Arnab Kumar De and Amaresh Dalal, "Numerical simulation of unconfined flow past a triangular cylinder," International Journal for Numerical Methods in Fluids 52, 801-821 (2006).

[51] J. B. Perot and C. J. Zusi, "Differential forms for scientists and engineers," Journal of Computational Physics 257, 1373-1393 (2014).

[52] Harley Flanders, Differential Forms with Applications to the Physical Sciences by Harley Flanders, Vol. 11 (Elsevier, 1963).

[53] J Blair Perot and V Subramanian, "Discrete calculus methods for diffusion," Journal of Computational Physics 224, 59-81 (2007).

[54] Ralph Abraham, Jerrold E Marsden, and Tudor Ratiu, Manifolds, tensor analysis, and applications, Vol. 75 (Springer Science \& Business Media, 2012).

[55] A. N. Hirani, Discrete exterior calculus, Ph.D. thesis, California Institute of Technology (2003).

[56] Mathieu Desbrun, Anil N Hirani, and Jerrold E Marsden, "Discrete exterior calculus for variational problems in computer vision and graphics," in Decision and Control, 2003. Proceedings. 42nd IEEE Conference on, Vol. 5 (IEEE, 2003) pp. 4902-4907.

[57] M. Desbrun, A. N. Hirani, M. Leok, and J. E. Marsden, "Discrete exterior calculus," arXiv preprint math/0508341 (2005).

[58] Eitan Grinspun, Mathieu Desbrun, Konrad Polthier, Peter Schröder, and Ari Stern, "Discrete differential geometry: an applied introduction," ACM SIGGRAPH Course 
7, 1-139 (2006).

[59] Anil N Hirani, Kalyana B Nakshatrala, and Jehanzeb H Chaudhry, "Numerical method for darcy flow derived using discrete exterior calculus," arXiv preprint arXiv:0810.3434 (2008).

[60] Mathieu Desbrun, Eva Kanso, and Yiying Tong, "Discrete differential forms for computational modeling," in Discrete differential geometry (Springer, 2008) pp. 287324.

[61] Keenan Crane, Fernando De Goes, Mathieu Desbrun, and Peter Schröder, "Digital geometry processing with discrete exterior calculus," in ACM SIGGRAPH 2013 Courses (ACM, 2013) p. 7.

[62] Fernando de Goes, Mathieu Desbrun, Mark Meyer, and Tony DeRose, "Subdivision exterior calculus for geometry processing," ACM Transactions on Graphics (TOG) 35, 133 (2016).

[63] I. Nitschke, S. Reuther, and A. Voigt, "Discrete exterior calculus (dec) for the surface navier-stokes equation," in Transport Processes at Fluidic Interfaces (Springer, 2017) pp. 177-197. 